\title{
Transient Analysis of Some Rewarded Markov Models Using Randomization with Quasistationarity Detection
}

\author{
Juan A. Carrasco, Senior Member, IEEE
}

\begin{abstract}
Rewarded homogeneous continuous-time Markov chain (CTMC) models can be used to analyze performance, dependability and performability attributes of computer and telecommunication systems. In this paper, we consider rewarded CTMC models with a reward structure including reward rates associated with states and two measures summarizing the behavior in time of the resulting reward rate random variable: the expected transient reward rate at time $t$ and the expected averaged reward rate in the time interval $[0, t]$. Computation of those measures can be performed using the randomization method, which is numerically stable and has good error control. However, for large stiff models, the method is very expensive. Exploiting the existence of a quasistationary distribution in the subset of transient states of discrete-time Markov chains with a certain structure, we develop a new variant of the (standard) randomization method, randomization with quasistationarity detection, covering finite CTMC models with state space $S \cup\left\{f_{1}, f_{2}, \ldots, f_{A}\right\}, A \geq 1$, where all states in $S$ are transient and reachable among them and the states $f_{i}$ are absorbing. The method has the same good properties as the standard randomization method and can be much more efficient. We also compare the performance of the method with that of regenerative randomization.
\end{abstract}

Index Terms-Rewarded continuous-time Markov chains, transient analysis, randomization, quasistationary distribution.

\section{INTRODUCTION}

$\mathrm{R}$ EWARDED homogeneous continuous-time Markov chain (CTMC) models can be used to analyze performance, dependability, and performability attributes of computer and telecommunication systems. A rewarded CTMC model is a CTMC $X=\{X(t) ; t \geq 0\}$ with a reward structure imposed over it. The reward structure may, in general, include reward rates $r_{i}$ associated with states and impulse rewards $r_{i, j}$ associated with transitions, where $r_{i}$ has the meaning of "rate" at which reward is earned while $X$ is in state $i$ and $r_{i, j}$ has the meaning of reward which is earned each time $X$ makes a transition from state $i$ to state $j$. In this paper, we will formally restrict our attention to finite rewarded CTMC models including only nonnegative reward rates and will consider two measures summarizing the behavior in time of the reward rate random variable $r_{X(t)}$ : the expected transient reward rate at time $t$, $\operatorname{ETRR}(t)=E\left[r_{X(t)}\right]$, and the expected averaged reward rate in the time interval $[0, t], E A R R(t)=E\left[\int_{0}^{t} r_{X(\tau)} d \tau / t\right]$. As examples of instances of the $\operatorname{ETRR}(t)$ and $\operatorname{EARR}(t)$ measures, consider a CTMC modeling a fault-tolerant system which can be up or down and assume that a reward rate 0 is assigned to the states in which the system is up and a reward rate 1 is assigned to the states in which the system is down. Then, $\operatorname{ETRR}(t)$ would be the unavailability of the

- The author is with the Departament d'Enginyeria Electrònica, Universitat Politècnica de Catalunya, Diagonal 647, plta. 9, 08028 Barcelona, Spain. E-mail: carrasco@eel.upc.es.

Manuscript received 17 Dec. 2002; revised 15 Jan. 2004; accepted 19 Jan. 2004.

For information on obtaining reprints of this article, please send e-mail to: tc@computer.org, and reference IEEECS Log Number 117979. system at time $t$ (i.e., the probability that the system is down at time $t$ ) and $\operatorname{EARR}(t)$ would be the expected interval unavailability at time $t$ (i.e., the expected value of the fraction of time that the system is down in the interval $[0, t]$ ). The assumption that all reward rates are nonnegative is taken to guarantee the stability of all randomization-based numerical methods we will consider. The assumption can, however, be easily circumvented by shifting in case some reward rate $r_{i}$ is $<0$, all reward rates by any positive quantity $d$ so that all new reward rates $r_{i}^{\prime}=r_{i}+d$ are $\geq 0$ : The $\operatorname{ETRR}(t)$ and $\operatorname{EARR}(t)$ measures of the original model are related to the corresponding measures, $\operatorname{ETRR}^{\prime}(t)$ and $E A R R^{\prime}(t)$, of the model with shifted reward rates by $\operatorname{ETRR}(t)=E T R R^{\prime}(t)-d$ and $E A R R(t)=E A R R^{\prime}(t)-d$. Also, by generalizing the definitions of $\operatorname{ETRR}(t)$ and $E A R R(t)$ as:

$$
\begin{aligned}
& \operatorname{ETRR}(t)=\lim _{\Delta t \rightarrow 0} \frac{E[\text { reward accumulated in }[t, t+\Delta t]]}{\Delta t}, \\
& \operatorname{EARR}(t)=E\left[\frac{\text { reward accumulated in }[0, t]}{t}\right],
\end{aligned}
$$

impulse rewards can be accomodated in the assumed framework by translating each nonnull impulse reward $r_{i, j}$ into a contribution $\lambda_{i, j} r_{i, j}$ to the reward rate associated with state $i, \lambda_{i, j}$ being the transition rate of $X$ from $i$ to $j$.

Computation of the $\operatorname{ETRR}(t)$ and $\operatorname{EARR}(t)$ measures requires the transient analysis of the CTMC. Commonly used methods to perform such analysis are ODE (ordinary differential equation) solvers and randomization. Good recent reviews of these methods with new results can be found in [16], [17], [23], [24]. The randomization method (also called uniformization) is attractive because of its 
excellent numerical stability and the fact that the computation error is well-controlled. It was first proposed by Grassman [11] and has been further developed by Gross and Miller [13] and Melamed and Yadin [18], [19]. The method is also offered by well-known performance, dependability, and performability modeling packages [2], [8], [10], [26]. The randomization method is based on the following result [14, Theorem 4.19]: Let $X=\{X(t) ; t \geq 0\}$ be a CTMC with finite state space $\Omega$; let $\lambda_{i, j}, i, j \in \Omega, i \neq j$, be the transition rate of $X$ from state $i$ to state $j$, and let $\lambda_{i}=\sum_{j \in \Omega-\{i\}} \lambda_{i, j}, i \in \Omega$ be the output rate of $X$ from state $i$. Consider any $\Lambda \geq \max _{i \in \Omega} \lambda_{i}$ and define the homogeneous discrete time Markov chain (DTMC) $\widehat{X}=\left\{\widehat{X}_{k} ; k=\right.$ $0,1,2, \ldots\}$ with the same state space and initial probability distribution as $X$ and transition probabilities

$$
P\left[\widehat{X}_{n+1}=j \mid \widehat{X}_{n}=i\right]=P_{i, j}=\lambda_{i, j} / \Lambda,
$$

$i, j \in \Omega, \quad i \neq j, \quad P\left[\widehat{X}_{n+1}=i \mid \widehat{X}_{n}=i\right]=P_{i, i}=1-\lambda_{i} / \Lambda, \quad i \in \Omega$. Let $Q=\{Q(t) ; t \geq 0\}$ be a Poisson process with arrival rate $\Lambda$ independent of $\widehat{X}$. Then, $X=\{X(t) ; t \geq 0\}$ is probabilistically identical to $\left\{\widehat{X}_{Q(t)} ; t \geq 0\right\}$. Next, we will review typical implementations of the randomization method for the computation of the $\operatorname{ETRR}(t)$ and $\operatorname{EARR}(t)$ measures.

We will start with the $\operatorname{ETRR}(t)$ measure. Using the facts that $X=\{X(t) ; t \geq 0\}$ and $\left\{\widehat{X}_{Q(t)} ; t \geq 0\right\}$ are probabilistically identical and that $Q$ and $\widehat{X}$ are independent, we can express $\operatorname{ETRR}(t)$ in terms of the transient regime of $\widehat{X}$ as

$$
\begin{aligned}
\operatorname{ETRR}(t) & =\sum_{i \in \Omega} r_{i} P[X(t)=i]=\sum_{i \in \Omega} r_{i} P\left[\widehat{X}_{Q(t)}=i\right] \\
& =\sum_{i \in \Omega} r_{i} \sum_{n=0}^{\infty} P\left[\widehat{X}_{n}=i \mid Q(t)=n\right] P[Q(t)=n] \\
& =\sum_{i \in \Omega} r_{i} \sum_{n=0}^{\infty} P\left[\widehat{X}_{n}=i\right] P[Q(t)=n] \\
& =\sum_{i \in \Omega} r_{i} \sum_{n=0}^{\infty} P\left[\widehat{X}_{n}=i\right] e^{-\Lambda t} \frac{(\Lambda t)^{n}}{n !} \\
& =\sum_{n=0}^{\infty} d(n) e^{-\Lambda t} \frac{(\Lambda t)^{n}}{n !},
\end{aligned}
$$

with $d(n)=\sum_{i \in \Omega} r_{i} P\left[\widehat{X}_{n}=i\right]$. Denoting by $\mathbf{q}(n)=\left(P\left[\widehat{X}_{n}=\right.\right.$ $i])_{i \in \Omega}$ the probability distribution (column) vector of $\widehat{X}$ at step $n, \mathbf{q}(n), n>0$ can be obtained from $\mathbf{q}(0)$ using

$$
\mathbf{q}(n+1)^{T}=\mathbf{q}(n)^{T} \mathbf{P},
$$

where $\mathbf{P}=\left(P_{i, j}\right)_{i, j \in \Omega}$ is the transition probability matrix of $\widehat{X}$ and $\mathbf{x}^{T}$ denotes the transpose of vector $\mathbf{x}$. In a typical implementation of the randomization method, an approximate value for $\operatorname{ETRR}(t), \operatorname{ETRR}_{N}^{a}(t)$, is obtained by truncating the infinite summation in (1) so that $N$ steps have to be given to $\widehat{X}$ :

$$
\operatorname{ETRR}_{N}^{a}(t)=\sum_{n=0}^{N} d(n) e^{-\Lambda t} \frac{(\Lambda t)^{n}}{n !},
$$

using $0 \leq d(n) \leq r_{\max }=\max _{i \in \Omega} r_{i}$, the truncation error is bounded as

$$
0 \leq \operatorname{ETRR}(t)-\operatorname{ETRR}_{N}^{a}(t) \leq r_{\max } \sum_{n=N+1}^{\infty} e^{-\Lambda t} \frac{(\Lambda t)^{n}}{n !},
$$

and, being $\varepsilon$ the requested absolute error, $N$ is chosen using

$$
N=\min \left\{m \geq 0: r_{\max } \sum_{n=m+1}^{\infty} e^{-\Lambda t} \frac{(\Lambda t)^{n}}{n !} \leq \varepsilon\right\} .
$$

We consider next the $\operatorname{EARR}(t)$ measure. Noting that

$$
\begin{aligned}
\operatorname{EARR}(t) & =E\left[\int_{0}^{t} r_{X(\tau)} d \tau / t\right]=\frac{1}{t} \int_{0}^{t} E\left[r_{X(\tau)}\right] d \tau \\
& =\frac{1}{t} \int_{0}^{t} \operatorname{ETRR}(\tau) d \tau
\end{aligned}
$$

and using (1) and

$$
\int_{0}^{t} e^{-\Lambda \tau}(\Lambda \tau)^{n} / n ! d \tau=\frac{1}{\Lambda} \sum_{m=n+1}^{\infty} e^{-\Lambda t}(\Lambda t)^{m} / m !
$$

we can express $E A R R(t)$ in terms of the transient regime of $\widehat{X}$ as

$$
\begin{aligned}
\operatorname{EARR}(t) & =\frac{1}{t} \sum_{n=0}^{\infty} d(n) \int_{0}^{t} e^{-\Lambda \tau} \frac{(\Lambda \tau)^{n}}{n !} d \tau \\
& =\frac{1}{\Lambda t} \sum_{n=0}^{\infty} d(n) \sum_{m=n+1}^{\infty} e^{-\Lambda t} \frac{(\Lambda t)^{m}}{m !} .
\end{aligned}
$$

In a typical implementation of the randomization method, an approximate value for $\operatorname{EARR}(t), \operatorname{EARR}_{N}^{a}(t)$, is obtained by truncating both summatories in the following way, which implies giving $N$ steps to $\widehat{X}$ :

$$
\begin{aligned}
\operatorname{EARR}_{N}^{a}(t) & =\frac{1}{\Lambda t} \sum_{n=0}^{N} d(n) \sum_{m=n+1}^{N+1} e^{-\Lambda t} \frac{(\Lambda t)^{m}}{m !} \\
& =\frac{1}{\Lambda t} \sum_{n=1}^{N+1} e^{-\Lambda t} \frac{(\Lambda t)^{n}}{n !} \sum_{m=0}^{n-1} d(m),
\end{aligned}
$$

using $0 \leq d(n) \leq r_{\max }$, the error is bounded as

$$
\begin{aligned}
0 \leq & \operatorname{EARR}(t)-\operatorname{EARR}_{N}^{a}(t)=\frac{1}{\Lambda t} \sum_{n=N+2}^{\infty} e^{-\Lambda t} \frac{(\Lambda t)^{n}}{n !} \sum_{m=0}^{n-1} d(m) \\
\leq & \frac{r_{\max }}{\Lambda t} \sum_{n=N+2}^{\infty} n e^{-\Lambda t} \frac{(\Lambda t)^{n}}{n !}=r_{\max } \sum_{n=N+1}^{\infty} e^{-\Lambda t} \frac{(\Lambda t)^{n}}{n !}
\end{aligned}
$$

and, $\varepsilon$ being the requested absolute error, $N$ is chosen using

$$
N=\min \left\{m \geq 0: r_{\max } \sum_{n=m+1}^{\infty} e^{-\Lambda t} \frac{(\Lambda t)^{n}}{n !} \leq \varepsilon\right\} .
$$

Alternative implementations with slightly different truncations have also been proposed [12].

Stable and efficient computation of the Poisson probabilities $e^{-\lambda} \lambda^{n} / n$ ! is a delicate issue and several approaches have been proposed [3], [9], [15], [22]. Our implementations of all randomization-based methods use the approach described in [15, pp. 1028-1029] (see also [1]).

For large models, the computational cost of the randomization method is roughly due to the $N$ vector-matrix multiplications (2). The truncation parameter $N$ increases 
with $\Lambda t$ and, for that reason, $\Lambda$ is usually taken equal to $\max _{i \in \Omega} \lambda_{i}$. Using the well-known result (see, for instance, [25, Theorem 3.3.5]) that $Q(t)$ has, for $\Lambda t \rightarrow \infty$, an asymptotic normal distribution with mean and variance $\Lambda t$, it is easy to realize that, for large $\Lambda t$ and $\varepsilon \ll 1$, the required $N$ will be $\approx \Lambda t$ and, if the model is large and stiff $\left(\max _{i \in \Omega} \lambda_{i} t\right.$ is a practical measure of stiffness), the randomization method will be expensive.

Several variants of the (standard) randomization method have been proposed to improve its efficiency. Miller has used selective randomization to solve reliability models with detailed representation of error handling activities [20]. The idea behind selective randomization [18] is to randomize the model only in a subset of the state space. Reibman and Trivedi [23] have proposed an approach based on the multistep concept. The idea is to compute $\mathbf{P}^{M}$ explicitly, where $M$ is the length of the multistep, and use the recurrence $\mathbf{q}(n+M)^{T}=\mathbf{q}(n)^{T} \mathbf{P}^{M}$ to advance $\widehat{X}$ faster for steps which have negligible contributions to the transient solution of $X$. Since, for large $\Lambda t$, the number of $\mathbf{q}(n)$ s with significant contributions is on the order of $\sqrt{\Lambda t}$, the multistep concept allows a significant reduction of the required number of vector-matrix multiplications when $\Lambda t$ is large. However, when computing $\mathbf{P}^{M}$, significant fill-in can occur if $\mathbf{P}$ is sparse. Adaptive uniformization [21] is a method in which the randomization rate is adapted depending on the states in which the randomized DTMC can be at a given step. Numerical experiments have shown that adaptive uniformization can be significantly faster than standard randomization for short to medium mission times. In addition, it can be used to solve models with infinite state spaces and not uniformly bounded output rates. More recently, the combination of adaptive and standard randomization to obtain a method which outperforms both for most models has been proposed [22]. A proposal to speed up the randomization method for irreducible models is steady-state detection [16]. A method based on steady-state detection with error bounds has been developed [27]. Regenerative randomization [4], [5] is another recently proposed randomization-based method. The method covers models with a certain structure and requires the selection of the so-called regenerative state. The performance of the method depends on that selection. For a class of models, class $C^{\prime}$, including typical failure/repair models with exponential failure and repair time distributions and repair in every state with failed components, a natural selection for the regenerative state exists and theoretical results are available, assessing the performance of the method for that natural selection in terms of visible model characteristics. Bounding regenerative randomization [6] is a variant of regenerative randomization which obtains bounds for reliability-like measures. For a class of models, class $C^{\prime \prime}$, including typical reliability-like failure/repair models with exponential failure and repair time distributions and repair in every state with failed components, the method is inexpensive and seems to provide tight bounds.

In this paper, we develop a new variant of the standard randomization method for the computation of the $\operatorname{ETRR}(t)$ and $\operatorname{EARR}(t)$ measures called randomization with quasistationarity detection. The method exploits the existence of a quasistationary distribution in the subset of transient states of DTMCs with a certain structure, has the same good properties as standard randomization (numerical stability and well-controlled computation error), and can be much more efficient. The rest of the paper is organized as follows: Section 2 describes the class of models covered by the method, reviews the theoretical results on which the method is based, and gives a first approximation to the method for the computation of the $\operatorname{ETRR}(t)$ meaasure without well-controlled computation error. Section 3 develops and describes the method in detail. Section 4 analyzes the performance of the method. We compare numerically the performance of the method with that of standard randomization and regenerative randomization for class $C^{\prime}$ models and with that of standard randomization for a model outside class $C^{\prime}$ for which regenerative randomization performs poorly. Finally, Section 5 presents the conclusions. The supplement (which can be found on the Computer Society Digital Library at http://computer.org/tc/archives.htm) includes some proofs and technical details.

\section{Preliminaries}

Randomization with quasistationarity detection covers finite CTMC models $X=\{X(t) ; t \geq 0\}$ with state space $\Omega=S \cup\left\{f_{1}, f_{2}, \ldots, f_{A}\right\}, A \geq 1$, where all states in $S$ are transient and reachable among them and the states $f_{i}$ are absorbing. We also assume $P[X(0) \in S]>0$ and that all states $f_{i}$ are reachable from $S$. The assumed class of CTMC models have important applications. Thus, $S$ could include the operational states of a fault-tolerant system and entry into the single absorbing state $f_{1}$ could model system failure; then, with a reward rate structure $r_{i}=0, i \in S$, and $r_{f_{1}}=1$, the $\operatorname{ETRR}(t)$ measure would be the unreliability of the system at time $t$. Bounding models are also covered. Those models are useful when an exact model would have an unmanageable size. A lower bounding unreliability model would have two absorbing states, $f_{1}, f_{2}, S$ would include a strict subset of the operational states, entry into $f_{1}$ would model system failure from a state in $S$, and entry into $f_{2}$ would model entry from $S$ into an operational state outside $S$; the model would have an initial probability distribution with same initial probabilities in $S$ as the "exact" model, an initial probability in $f_{1}$ equal to the probability that initially the system has failed, and an initial probability in $f_{2}$ equal to the probability that initially the system is in an operational state outside $S$; then, with a reward rate structure $r_{i}=0, i \in S, r_{f_{1}}=1$, and $r_{f_{2}}=0$, the $\operatorname{ETRR}(t)$ measure would be a lower bound for the unreliability of the system at time $t$. An upper bounding unreliability model would have a single absorbing state, $f_{1}$, $S$ would include a strict subset of the operational states, and entry into $f_{1}$ would model either system failure from a state in $S$ or entry from $S$ into an operational state outside $S$; the model would have an initial probability distribution with initial probabilities in $S$ as the "exact" model and initial probability in $f_{1}$ equal to the probability that, initially, the system either has failed or is in an operational state outside $S$; then, with a reward rate structure $r_{i}=0, i \in S, r_{f_{1}}=1$, the $\operatorname{ETRR}(t)$ measure would be an upper bound for the unreliability of the system at time $t$. Bounding models with 


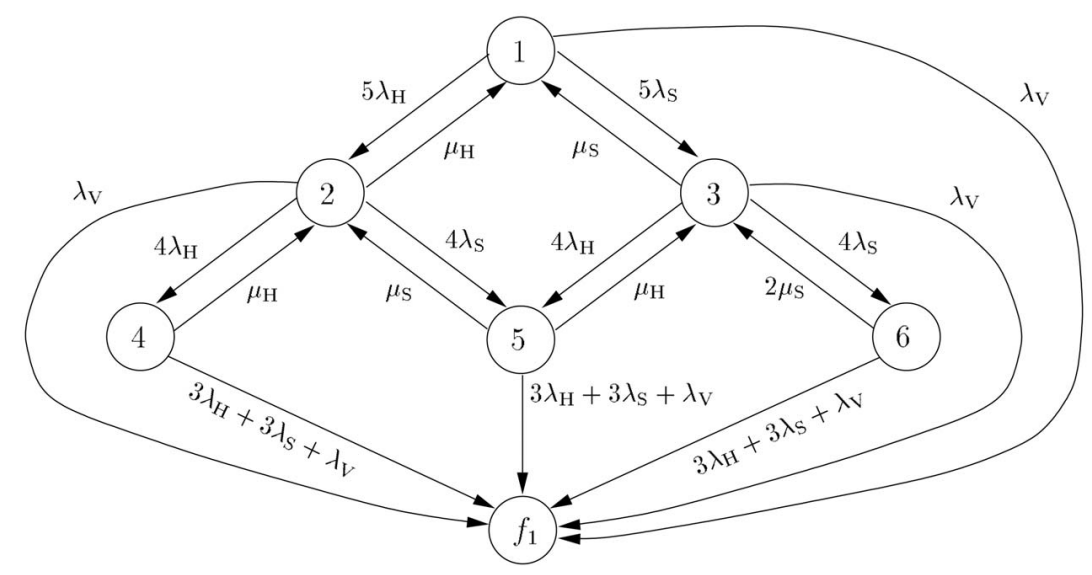

Fig. 1. Small unreliability model corresponding to a repairable fault-tolerant system using the NMR redundancy technique and having an imperfect voter.

more general reward rate structures allow the computation of bounds for the expected transient reward rate and the expected averaged reward rate measures of models of unmanageable size. In a lower bounding model of that type, $S$ would include a strict subset of the state space of the exact model and entry into a single absorbing state $f_{1}$ would model exit from $S$; the model would have an initial probability distribution with initial probabilities in $S$ as the "exact" model and an initial probability in $f_{1}$ equal to the probability that, initially, the system is in a state outside $S$; then, assigning to the states in $S$ the same reward rates as in the exact model and assigning to $f_{1}$ a lower bound for the reward rate of any state of the exact model, the $\operatorname{ETRR}(t)$ and $\operatorname{EARR}(t)$ measures of the bounding model would lower bound the corresponding measures of the exact model. In an upper bounding model, the only difference would be that the reward rate assigned to $f_{1}$ would have to upper bound the reward rate of any state of the exact model and the $\operatorname{ETRR}(t)$ and $\operatorname{EARR}(t)$ measures of the bounding model would upper bound the corresponding measures of the exact model.

As an illustration of the CTMC models covered by randomization with quasistationarity detection, Fig. 1 gives a small unreliability model corresponding to a repairable fault-tolerant system using the NMR redundancy technique with five processing modules and an imperfect voter in which processing modules fail in "hard" mode with rate $\lambda_{\mathrm{H}}$ and in "soft" mode with rate $\lambda_{\mathrm{S}}$ and the voter fails with rate $\lambda_{\mathrm{V}}$. It is assumed that the system fails when either the voter fails or a processing module fails when only three processing modules are unfailed and that, initially, no component is failed. Processing modules in hard failure are repaired by a single repairman with rate $\mu_{\mathrm{H}}$ and processing modules in soft failure are repaired by an unlimited number of repairmen with rate $\mu_{\mathrm{S}}$. With a reward rate structure $r_{i}=0, i \in\{1,2,3,4,5,6\}$ and $r_{f_{1}}=1$, the $\operatorname{ETRR}(t)$ measure is the unreliability of the fault-tolerant system at time $t$. The model belongs to the class covered by randomization with quasistationarity detection with $S=\{1,2,3,4,5,6\}$.

The randomization with quasistationarity detection method exploits the following result (see, for instance, [14, Section 2.8]).
Theorem 1. Let $\mathbf{Q}$ be the restriction to $B \times B$, where $B$ is the finite subset of transient states of a DTMC $Y=\left\{Y_{n} ; n=0,1,2, \ldots\right\}$, of the transition probability matrix of $Y$, assume that $\mathbf{Q}$ is regular, ${ }^{1}$ let $\beta$ be the (nonnull) initial probability distribution vector of $Y$ restricted to $B$, and let $\mathbf{1}$ be $a|B|$-vector with all its components equal to 1 . Then,

$$
\lim _{n \rightarrow \infty} \frac{\beta^{T} \mathbf{Q}^{n}}{\beta^{T} \mathbf{Q}^{n} \mathbf{1}}=\mathbf{u}^{T}
$$

where $\mathbf{u}$ is the normalized $\left(\mathbf{u}^{T} \mathbf{1}=1\right)$ Perron-Frobenius left eigenvector of $\mathbf{Q}$.

Theorem 1 has a probabilistic interpretation: $\beta^{T} \mathbf{Q}^{n}$ is the probability distribution row vector of $Y$ in the subset of transient states $B$ and, then, $\beta^{T} \mathbf{Q}^{n} /\left(\beta^{T} \mathbf{Q}^{n} \mathbf{1}\right)$ is the conditional probability distribution row vector of $Y$ at step $n$ in the subset of transient states $B$. The theorem states that that conditional probability distribution has a limit which is independent of the initial probability distribution of $Y$ in $B$ and is equal to the normalized Perron-Frobenius left eigenvector, with all its components strictly positive, of the restriction of the transition probability matrix of $Y$ to $B \times B$. The limit is called quasistationary distribution because, as is easy to prove from $\mathbf{u}^{T} \mathbf{Q}=\gamma \mathbf{u}^{T}$ and $\mathbf{u}^{T} \mathbf{1}=1, \gamma$ being the spectral radius of $\mathbf{Q}$ (eigenvalue associated with $\mathbf{u}$ ),

$$
\frac{\mathbf{u}^{T} \mathbf{Q}}{\mathbf{u}^{T} \mathbf{Q} \mathbf{1}}=\mathbf{u}^{T}
$$

which implies that, if $Y$ has, at some step, a conditional probability distribution in $B$ equal to $\mathbf{u}$, it will have that conditional probability distribution in $B$ at successive steps. An immediate consequence of Theorem 1 is the following result:

Corollary 1. Let $\mathbf{Q}$ be the restriction to $B \times B$, where $B$ is the finite subset of transient states of a DTMC $Y=\left\{Y_{n} ; n=0,1,2, \ldots\right\}$, of the transition probability matrix of $Y$, assume that $\mathbf{Q}$ is regular, let $\beta$ be the (nonnull) initial probability distribution

1. A nonnegative square matrix $\mathbf{A}$ is said to be regular (primitive) if all elements of $\mathbf{A}^{k}$ are $>0$ for some positive integer $k$ (and, therefore, for all sufficiently large $k$ ). 
vector of $Y$ restricted to $B$, let $\mathbf{1}$ be a $|B|$-vector with all its components equal to 1 , and let $\mathbf{z}=\left(z_{i}\right)_{i \in B}$ be an arbitrary $|B|$-vector. Then,

$$
\lim _{n \rightarrow \infty} \frac{\boldsymbol{\beta}^{T} \mathbf{Q}^{n} \mathbf{z}}{\boldsymbol{\beta}^{T} \mathbf{Q}^{n} \mathbf{1}}=\mathbf{u}^{T} \mathbf{z}
$$

where $\mathbf{u}$ is the normalized $\left(\mathbf{u}^{T} \mathbf{1}=1\right)$ Perron-Frobenius left eigenvector of $\mathbf{Q}$.

Corollary 1 also has a probabilistic interpretation: $\boldsymbol{\beta}^{T} \mathbf{Q}^{n} \mathbf{z} /\left(\boldsymbol{\beta}^{T} \mathbf{Q}^{n} \mathbf{1}\right)$ is the expected value of $z_{Y_{n}}$ conditioned to $Y_{n} \in B$ and, then, Corollary 1 states that that expected value has a limit which is independent of the initial probability distribution of $Y$ in $B$. Corollary 1 is the basis of the randomization with quasistationarity detection method. We will give, in the remainder of this section, a first approximation to the method for the $\operatorname{ETRR}(t)$ measure in which the error is not well-controlled.

Let $\lambda_{i, j}, i \in \Omega, j \in \Omega-\{i\}$, and $\lambda_{i}$ denote, respectively, the transition rates and output rates of $X$ and let $\alpha_{i}=P[X(0)=i], i \in \Omega$. Let $\widehat{X}$ be the randomized DTMC of $X$ under a randomization rate $\Lambda$ slightly larger than $\max _{i \in S} \lambda_{i}$, say $\Lambda=(1+\theta) \max _{i \in S} \lambda_{i}, \quad \theta=10^{-4}$. Let $\mathbf{P}=\left(P_{i, j}\right)_{i, j \in \Omega}$, $P_{i, j}=\lambda_{i, j} / \Lambda, \quad i, j \in \Omega, i \neq j, P_{i, i}=1-\lambda_{i} / \Lambda, i \in \Omega$, be the transition probability matrix of $\widehat{X}$. Let $\mathbf{P}_{S, S}=\left(P_{i, j}\right)_{i, j \in S}$ and let $\alpha_{S}=\left(\alpha_{i}\right)_{i \in S}$. Also, given $B \subset \Omega$, let $P_{i, B}=\sum_{j \in B} P_{i, j}$ and $\alpha_{B}=\sum_{i \in B} \alpha_{i}$. Since the states in $S$ are reachable among them in $\widehat{X}$ (because they are reachable among them in $X$ ), $\mathbf{P}_{S, S}$ is irreducible. This, together with $P_{i, i}>0, i \in S$, implies that $\mathbf{P}_{S, S}$ is regular. Then, letting $\mathbf{1}$ be an $|S|$-vector with all its components equal to 1 , since $S$ is the subset of transient states of $\widehat{X}$, according to Corollary 1 , for any $|S|$-vector $\mathbf{z}=\left(z_{j}\right)_{j \in S}$,

$$
z(n)=E\left[z_{\widehat{X}_{n}} \mid \widehat{X}_{n} \in S\right]=\frac{\boldsymbol{\alpha}_{S}^{T} \mathbf{P}_{S, S}^{n} \mathbf{z}}{\boldsymbol{\alpha}_{S}^{T} \mathbf{P}_{S, S}^{n} \mathbf{1}}
$$

has limit for $n \rightarrow \infty$. Let

$$
\begin{aligned}
\mathbf{r} & =\left(r_{j}\right)_{j \in S}, \\
\mathbf{w} & =\left(w_{j}\right)_{j \in S}=\left(P_{j, S}\right)_{j \in S}, \\
\mathbf{v}^{i} & =\left(v_{j}^{i}\right)_{j \in S}=\left(P_{j, f_{i}}\right)_{j \in S}, \quad 1 \leq i \leq A, \\
q_{j}(n) & =P\left[\widehat{X}_{n}=j\right], j \in \Omega,
\end{aligned}
$$

and let

$$
\begin{gathered}
r(n)=E\left[r_{\widehat{X}_{n}} \mid \widehat{X}_{n} \in S\right]=\frac{\boldsymbol{\alpha}_{S}^{T} \mathbf{P}_{S, S}^{n} \mathbf{r}}{\boldsymbol{\alpha}_{S}^{T} \mathbf{P}_{S, S}^{n} \mathbf{1}}=\frac{\sum_{j \in S} q_{j}(n) r_{j}}{\sum_{j \in S} q_{j}(n)}, \\
w(n)=E\left[w_{\widehat{X}_{n}} \mid \widehat{X}_{n} \in S\right]=\frac{\boldsymbol{\alpha}_{S}^{T} \mathbf{P}_{S, S}^{n} \mathbf{w}}{\boldsymbol{\alpha}_{S}^{T} \mathbf{P}_{S, S}^{n} \mathbf{1}}=\frac{\sum_{j \in S} q_{j}(n) P_{j, S}}{\sum_{j \in S} q_{j}(n)}, \\
v^{i}(n)=E\left[v_{\widehat{X}_{n}}^{i} \mid \widehat{X}_{n} \in S\right]=\frac{\boldsymbol{\alpha}_{S}^{T} \mathbf{P}_{S, S}^{n} \mathbf{v}^{i}}{\boldsymbol{\alpha}_{S}^{T} \mathbf{P}_{S, S}^{n} \mathbf{1}}=\frac{\sum_{j \in S} q_{j}(n) P_{j, f_{i}}}{\sum_{j \in S} q_{j}(n)}, \\
1 \leq i \leq A
\end{gathered}
$$

Then, the sequences $r(n), w(n)$, and $v^{i}(n), 1 \leq i \leq A$ are convergent. The following proposition gives a formulation of the sequence $d(n)=\sum_{j \in \Omega} q_{j}(n) r_{j}, n=0,1,2, \ldots$ in terms of the sequences $r(n), w(n)$, and $v^{i}(n), 1 \leq i \leq A$, $n=0,1,2, \ldots$.

Proposition 1. For $n \geq 0$,

$$
d(n)=\nu(n) r(n)+\sum_{i=1}^{A} \alpha_{f_{i}} r_{f_{i}}+\sum_{m=0}^{n-1} \nu(m) \sum_{i=1}^{A} v^{i}(m) r_{f_{i}}
$$

with

$$
\nu(n)=\alpha_{S} \prod_{m=0}^{n-1} w(m)
$$

Proof. Let $\widehat{X}_{l: m} c$ denote the predicate which is true when $\widehat{X}_{k}$ satisfies condition $c$ for all $k, l \leq k \leq m$. Since $f_{i}$ is absorbing and, for $m \geq 0, \widehat{X}_{m} \in S$ if and only if $\widehat{X}_{0: m} \in S$ :

$$
\begin{aligned}
q_{f_{i}}(n)= & P\left[\widehat{X}_{n}=f_{i}\right]=P\left[\widehat{X}_{0}=f_{i}\right] \\
& +\sum_{m=0}^{n-1} P\left[\widehat{X}_{0: m} \in S \wedge \widehat{X}_{m+1}=f_{i}\right] \\
= & P\left[\widehat{X}_{0}=f_{i}\right]+\sum_{m=0}^{n-1} P\left[\widehat{X}_{m} \in S \wedge \widehat{X}_{m+1}=f_{i}\right] \\
= & \alpha_{f_{i}}+\sum_{m=0}^{n-1} \sum_{j \in S} q_{j}(m) P_{j, f_{i}} .
\end{aligned}
$$

Define $\nu(n)=\sum_{j \in S} q_{j}(n)$. From (6),

$$
\begin{aligned}
q_{f_{i}}(n) & =\alpha_{f_{i}}+\sum_{m=0}^{n-1} \sum_{k \in S} q_{k}(m) \frac{\sum_{j \in S} q_{j}(m) P_{j, f_{i}}}{\sum_{j \in S} q_{j}(m)} \\
& =\alpha_{f_{i}}+\sum_{m=0}^{n-1} \nu(m) v^{i}(m) .
\end{aligned}
$$

Then,

$$
\begin{aligned}
d(n)= & \sum_{j \in S} q_{j}(n) r_{j}+\sum_{i=1}^{A} q_{f_{i}}(n) r_{f_{i}} \\
= & \sum_{k \in S} q_{k}(n) \frac{\sum_{j \in S} q_{j}(n) r_{j}}{\sum_{j \in S} q_{j}(n)} \\
& \quad+\sum_{i=1}^{A} \alpha_{f_{i}} r_{f_{i}}+\sum_{m=0}^{n-1} \nu(m) \sum_{i=1}^{A} v^{i}(m) r_{f_{i}} \\
= & \nu(n) r(n)+\sum_{i=1}^{A} \alpha_{f_{i}} r_{f_{i}}+\sum_{m=0}^{n-1} \nu(m) \sum_{i=1}^{A} v^{i}(m) r_{f_{i}} .
\end{aligned}
$$

On the other hand, since $\widehat{X}_{n} \in S$ if and only if $\widehat{X}_{0: n} \in S$ :

$$
\begin{aligned}
\nu(n) & =\sum_{j \in S} q_{j}(n)=P\left[\widehat{X}_{n} \in S\right]=P\left[\widehat{X}_{0: n} \in S\right] \\
& =P\left[\widehat{X}_{0} \in S\right] \prod_{m=0}^{n-1} P\left[\widehat{X}_{m+1} \in S \mid \widehat{X}_{0: m} \in S\right] \\
& =P\left[\widehat{X}_{0} \in S\right] \prod_{m=0}^{n-1} P\left[\widehat{X}_{m+1} \in S \mid \widehat{X}_{m} \in S\right]
\end{aligned}
$$




$$
\begin{aligned}
& =\alpha_{S} \prod_{m=0}^{n-1} \frac{P\left[\widehat{X}_{m} \in S \wedge \widehat{X}_{m+1} \in S\right]}{P\left[\widehat{X}_{m} \in S\right]}=\alpha_{S} \prod_{m=0}^{n-1} \frac{\sum_{j \in S} q_{j}(m) P_{j, S}}{\sum_{j \in S} q_{j}(m)} \\
& =\alpha_{S} \prod_{m=0}^{n-1} w(m) .
\end{aligned}
$$

The formulation of the sequence $d(n), n=0,1,2, \ldots$, in terms of the sequences $r(n), w(n)$, and $v^{i}(n), 1 \leq i \leq A$, $n=0,1,2, \ldots$, given by Proposition 1 , together with $\operatorname{ETRR}(t)=\sum_{n=0}^{\infty} d(n) e^{-\Lambda t}(\Lambda t)^{n} / n$ ! (see Section 1) gives the formulation of $\operatorname{ETRR}(t)$ in terms of the sequences $r(n), w(n)$, and $v^{i}(n), 1 \leq i \leq A, n=0,1,2, \ldots$, on which the randomization with quasistationarity detection method for the $\operatorname{ETRR}(t)$ measure is based. Using the fact that those sequences are convergent, we could obtain an approximate value for $\operatorname{ETRR}(t)$ by computing the sequences up to a value $K$ of $n$ for which the sequences "stabilize" with high accuracy and using the formulation with the values of the convergent sequences for $n>K$ substituted by the last computed element of the sequences. Those sequences could be computed from $\mathbf{q}(n)=\left(q_{j}(n)\right)_{j \in S}$ using $r(n)=\mathbf{q}(n)^{T} \mathbf{r} /\left(\mathbf{q}(n)^{T} \mathbf{1}\right), w(n)=\mathbf{q}(n)^{T} \mathbf{w} /\left(\mathbf{q}(n)^{T} \mathbf{1}\right)$, and $v^{i}(n)=\mathbf{q}(n)^{T} \mathbf{v}^{i} /\left(\mathbf{q}(n)^{T} \mathbf{1}\right), 1 \leq i \leq A$, where $\mathbf{q}(n), 0 \leq$ $n \leq K$ could be computed recursively using $\mathbf{q}(0)=\alpha_{S}$ and $\mathbf{q}(k+1)^{T}=\mathbf{q}(k)^{T} \mathbf{P}_{S, S}, \quad 0 \leq k \leq K-1$. However, that approach would not upper bound the error and, therefore, the resulting computational scheme would not have the same quality as standard randomization.

\section{The Method}

In this section, we develop and describe the randomization with quasistationarity detection method for the measures $\operatorname{ETRR}(t)$ and $\operatorname{EARR}(t)$. We will assume $t>0$ (for $\left.t=0, \operatorname{ETRR}(t)=E A R R(t)=E\left[r_{X(0)}\right]=\sum_{i \in \Omega} \alpha_{i} r_{i}\right)$. In the method, the sequences $r(n), w(n)$, and $v^{i}(n), 1 \leq i \leq A$, are computed up to some index $n=K$ and bounds for the $\operatorname{ETRR}(t)$ and $\operatorname{EARR}(t)$ measures are computed which become monotonically arbitrarily tight as $K$ increases. Then, by choosing a large enough $K$, the measures can be computed with arbitrary accuracy, ignoring, of course, roundoff errors, which should have a very small relative value since the method has excellent numerical stability. We start by deriving bounds for the elements of a sequence $z(n)=\boldsymbol{\alpha}_{S}^{T} \mathbf{P}_{S, S}^{n} \mathbf{z} /\left(\boldsymbol{\alpha}_{S}^{T} \mathbf{P}_{S, S}^{n} \mathbf{1}\right)$ with $n$ greater than some index $K \geq 0$, where $\mathbf{z}=\left(z_{j}\right)_{j \in S}$ is an arbitrary $|S|$-vector. We will use the following lemma.

Lemma 1. Let a be an n-vector with nonnegative components and some strictly positive component, let $\mathbf{b}$ be an arbitrary n-vector, and let $\mathbf{c}$ be an n-vector with strictly positive components. Then,

$$
\min _{1 \leq j \leq n} \frac{b_{j}}{c_{j}} \leq \frac{\mathbf{a}^{T} \mathbf{b}}{\mathbf{a}^{T} \mathbf{c}} \leq \max _{1 \leq j \leq n} \frac{b_{j}}{c_{j}} .
$$

Proof. See the Supplement (which can be found on the Computer Society Digital Library at http://computer. org/tc/archives.htm).

Let

$$
\mathbf{o}(n)=\left(o_{j}(n)\right)_{j \in S}=\mathbf{P}_{S, S}^{n} \mathbf{1},
$$

where $\mathbf{1}$ is an $|S|$-vector with all components equal to 1 . The bounds are given by the following theorem.

Theorem 2. Let $\mathbf{z}(n)=\left(z_{j}(n)\right)_{j \in S}=\mathbf{P}_{S, S}^{n} \mathbf{z}$ and let $z(n)=\boldsymbol{\alpha}_{S}^{T} \mathbf{P}_{S, S} \mathbf{z} /\left(\boldsymbol{\alpha}_{S}^{T} \mathbf{P}_{S, S}^{n} \mathbf{1}\right)$, where $\mathbf{z}=\left(z_{j}\right)_{j \in S}$ is an arbitrary $|S|$-vector. Then, for $n>K \geq 0$,

$$
\min _{j \in S} z_{j}(K) / o_{j}(K) \leq z(n) \leq \max _{j \in S} z_{j}(K) / o_{j}(K) .
$$

Proof. For $n>K$,

$$
z(n)=\frac{\boldsymbol{\alpha}_{S}^{T} \mathbf{P}_{S, S}^{n} \mathbf{z}}{\boldsymbol{\alpha}_{S}^{T} \mathbf{P}_{S, S}^{n} \mathbf{1}}=\frac{\boldsymbol{\alpha}_{S}^{T} \mathbf{P}_{S, S}^{n-K} \mathbf{P}_{S, S}^{K} \mathbf{z}}{\boldsymbol{\alpha}_{S}^{T} \mathbf{P}_{S, S}^{n-K} \mathbf{P}_{S, S}^{K} \mathbf{1}}=\frac{\boldsymbol{\alpha}_{S}^{T} \mathbf{P}_{S, S}^{n-K} \mathbf{z}(K)}{\boldsymbol{\alpha}_{S}^{T} \mathbf{P}_{S, S}^{n-K} \mathbf{o}(K)} .
$$

But, since $\mathbf{P}_{S, S}$ is irreducible, each row of $\mathbf{P}_{S, S}^{K}$ has at least some nonnull element and, therefore, every component of $\mathbf{o}(K)=\mathbf{P}_{S, S}^{K} \mathbf{1}$ is strictly positive. Also, since each row of $\mathbf{P}_{S, S}^{n-K}$ has at least some nonnull element and at least some $\alpha_{j}, j \in S$ is $>0$, the row vector $\boldsymbol{\alpha}_{S}^{T} \mathbf{P}_{S, S}^{n-K}$ has at least some strictly positive element and, then, the result follows from Lemma 1 with $\mathbf{a}^{T}=\boldsymbol{\alpha}_{S}^{T} \mathbf{P}_{S, S}^{n-K}, \mathbf{b}=\mathbf{z}(K)$, and $\mathbf{c}=\mathbf{o}(K)$.

The following theorem establishes that the bounds given by Theorem 2 become monotonically arbitrarily tight as $K \rightarrow \infty$.

Theorem 3. Let $\mathbf{u}$ be the Perron-Frobenius left eigenvector of $\mathbf{P}_{S, S}$. Let $\mathbf{z}(n)=\left(z_{j}(n)\right)_{j \in S}=\mathbf{P}_{S, S}^{n} \mathbf{z}$, where $\mathbf{z}=\left(z_{j}\right)_{j \in S}$ is an arbitrary $|S|$-vector. Let $K \geq 0$. Then,

1.

$$
\lim _{K \rightarrow \infty} \min _{j \in S} \frac{z_{j}(K)}{o_{j}(K)}=\lim _{K \rightarrow \infty} \max _{j \in S} \frac{z_{j}(K)}{o_{j}(K)}=\mathbf{u}^{T} \mathbf{z} .
$$

2. For $K^{\prime}>K$,

$$
\begin{aligned}
& \min _{j \in S} \frac{z_{j}(K)}{o_{j}(K)} \leq \min _{j \in S} \frac{z_{j}\left(K^{\prime}\right)}{o_{j}\left(K^{\prime}\right)} \\
& \leq \max _{j \in S} \frac{z_{j}\left(K^{\prime}\right)}{o_{j}\left(K^{\prime}\right)} \leq \max _{j \in S} \frac{z_{j}(K)}{o_{j}(K)} .
\end{aligned}
$$

Proof. Part 1. Let $\mathbf{e}_{j}$ denote an $|S|$-vector with component associated with $j \in S$ equal to 1 and all other components equal to 0 . We have $z_{j}(K)=\mathbf{e}_{j}^{T} \mathbf{z}(K), o_{j}(K)=\mathbf{e}_{j}^{T} \mathbf{o}(K)$, and

$$
\frac{z_{j}(K)}{o_{j}(K)}=\frac{\mathbf{e}_{j}^{T} \mathbf{z}(K)}{\mathbf{e}_{j}^{T} \mathbf{o}(K)}=\frac{\mathbf{e}_{j}^{T} \mathbf{P}_{S, S}^{K} \mathbf{z}}{\mathbf{e}_{j}^{T} \mathbf{P}_{S, S}^{K} \mathbf{1}} .
$$

Using Corollary 1 with $\beta=\mathbf{e}_{j}$ and $\mathbf{Q}=\mathbf{P}_{S, S}$,

$$
\lim _{K \rightarrow \infty} \frac{z_{j}(K)}{o_{j}(K)}=\mathbf{u}^{T} \mathbf{z},
$$

which implies the result. 
Part 2. Let $i \in S$. Using the same notation as in the proof of part 1, we have

$$
\begin{aligned}
\frac{z_{i}\left(K^{\prime}\right)}{o_{i}\left(K^{\prime}\right)} & =\frac{\mathbf{e}_{i}^{T} \mathbf{z}\left(K^{\prime}\right)}{\mathbf{e}_{i}^{T} \mathbf{o}\left(K^{\prime}\right)}=\frac{\mathbf{e}_{i}^{T} \mathbf{P}_{S, S}^{K^{\prime}} \mathbf{z}}{\mathbf{e}_{i}^{T} \mathbf{P}_{S, S}^{K^{\prime}} \mathbf{1}} \\
& =\frac{\mathbf{e}_{i}^{T} \mathbf{P}_{S, S}^{K^{\prime}-K} \mathbf{P}_{S, S}^{K} \mathbf{z}}{\mathbf{e}_{i}^{T} \mathbf{P}_{S, S}^{K^{\prime}-K} \mathbf{P}_{S, S}^{K} \mathbf{1}}=\frac{\mathbf{e}_{i}^{T} \mathbf{P}_{S, S}^{K^{\prime}-K} \mathbf{z}(K)}{\mathbf{e}_{i}^{T} \mathbf{P}_{S, S}^{K^{\prime}-K} \mathbf{o}(K)},
\end{aligned}
$$

which, every component of $\mathbf{o}(K)$ being strictly positive, (see the proof of Theorem 2) and having the row vector $\mathbf{e}_{i}^{T} \mathbf{P}_{S, S}^{K^{\prime}-K}$ at least some strictly positive element, which can be argued as it was argued in the proof of Theorem 2 that result for the row vector $\boldsymbol{\alpha}_{S}^{T} \mathbf{P}_{S, S}^{n-K}$, implies, by Lemma 1,

$$
\min _{j \in S} \frac{z_{j}(K)}{o_{j}(K)} \leq \frac{z_{i}\left(K^{\prime}\right)}{o_{i}\left(K^{\prime}\right)} \leq \max _{j \in S} \frac{z_{j}(K)}{o_{j}(K)},
$$

which implies the result.

Let

$$
\begin{gathered}
\mathbf{r}(n)=\left(r_{j}(n)\right)_{j \in S}=\mathbf{P}_{S, S}^{n} \mathbf{r}, \\
\mathbf{w}(n)=\left(w_{j}(n)\right)_{j \in S}=\mathbf{P}_{S, S}^{n} \mathbf{w},
\end{gathered}
$$

and

$$
\mathbf{v}^{i}(n)=\left(v_{j}^{i}(n)\right)_{j \in S}=\mathbf{P}_{S, S}^{n} \mathbf{v}^{i}, \quad 1 \leq i \leq A .
$$

Using Theorem 2,

$$
\begin{gathered}
r_{\mathrm{lb}}(K)=\min _{j \in S} \frac{r_{j}(K)}{o_{j}(K)} \leq r(n) \leq \max _{j \in S} \frac{r_{j}(K)}{o_{j}(K)}=r_{\mathrm{ub}}(K), \\
n>K \geq 0, \\
w_{\mathrm{lb}}(K)=\min _{j \in S} \frac{w_{j}(K)}{o_{j}(K)} \leq w(n) \leq \max _{j \in S} \frac{w_{j}(K)}{o_{j}(K)}=w_{\mathrm{ub}}(K), \\
n>K \geq 0, \\
v_{\mathrm{lb}}^{i}(K)=\min _{j \in S} \frac{v_{j}^{i}(K)}{o_{j}(K)} \leq v^{i}(n) \leq \max _{j \in S} \frac{v_{j}^{i}(K)}{o_{j}(K)}=v_{\mathrm{ub}}^{i}(K), \\
n>K \geq 0, \quad 1 \leq i \leq A .
\end{gathered}
$$

Let $K \geq 0$. Bounds for $d(n), n>K$, can be obtained from $w(m)$ and $v^{i}(m), 1 \leq i \leq A, m=0,1,2, \ldots, K$, and the bounds $r_{\mathrm{lb}}(K), r_{\mathrm{ub}}(K), w_{\mathrm{lb}}(K), w_{\mathrm{ub}}(K), v_{\mathrm{lb}}^{i}(K), v_{\mathrm{ub}}^{i}(K), 1 \leq$ $i \leq A$ for the elements of the sequences $r(n), w(n)$, and $v^{i}(n), 1 \leq i \leq A$, with $n>K$ given by (11)-(13) using the formulation for $d(n)$ given by Proposition 1 . The bounds are given by the following proposition, where $I_{c}$ denotes the indicator function returning the value 1 if condition $c$ is satisfied and the value 0 otherwise. Note that $P_{i, i}>0, i \in S$ implies $P_{i, S}>0$. In addition, since all states in $S$ are transient in $\widehat{X}, P_{i, S}<1$ for some state $i \in S$. Then, $\mathbf{w}(0)=$ $\mathbf{w}=\left(P_{i, S}\right)_{i \in S}$ has all components $>0$ and $\leq 1$ and some component $<1$. This implies $0<w_{\mathrm{lb}}(0)<1$ and $0<$ $w_{\mathrm{ub}}(0) \leq 1$ and, using Theorem $3,0<w_{\mathrm{lb}}(K)<1$ and $0<$ $w_{\mathrm{ub}}(K) \leq 1$ for all $K \geq 0$.

Proposition 2. For $n>K \geq 0, d_{\mathrm{lb}}^{K}(n) \leq d(n) \leq d_{\mathrm{ub}}^{K}(n)$, where

$$
\begin{aligned}
d_{\mathrm{lb}}^{K}(n) & =\sum_{i=1}^{A} \alpha_{f_{i}} r_{f_{i}}+\sum_{m=0}^{K} \nu(m) \sum_{i=1}^{A} v^{i}(m) r_{f_{i}} \\
& +\nu(K+1) r_{\mathrm{lb}}(K) w_{\mathrm{lb}}(K)^{n-K-1} \\
& +\nu(K+1)\left(\sum_{i=1}^{A} v_{\mathrm{lb}}^{i}(K) r_{f_{i}}\right) \frac{1-w_{\mathrm{lb}}(K)^{n-K-1}}{1-w_{\mathrm{lb}}(K)}, \\
d_{\mathrm{ub}}^{K}(n) & =\sum_{i=1}^{A} \alpha_{f_{i}} r_{f_{i}}+\sum_{m=0}^{K} \nu(m) \sum_{i=1}^{A} v^{i}(m) r_{f_{i}} \\
& +\nu(K+1) r_{\mathrm{ub}}(K) w_{\mathrm{ub}}(K)^{n-K-1} \\
& +\nu(K+1)\left(\sum_{i=1}^{A} v_{\mathrm{ub}}^{i}(K) r_{f_{i}}\right) \\
& \left(I_{w_{\mathrm{ub}}(K)<1} \frac{1-w_{\mathrm{ub}}(K)^{n-K-1}}{1-w_{\mathrm{ub}}(K)}+I_{w_{\mathrm{ub}}(K)=1}(n-K-1)\right), \\
\nu(m) & =\alpha_{S} \prod_{l=0}^{m-1} w(l),
\end{aligned}
$$

and $r_{\mathrm{lb}}(K), r_{\mathrm{ub}}(K), w_{\mathrm{lb}}(K), w_{\mathrm{ub}}(K)$ and $v_{\mathrm{lb}}^{i}(K), v_{\mathrm{ub}}^{i}(K)$, $1 \leq i \leq A$, are given by (11)-(13).

Proof. $d(n)$ (see Proposition 1) is increasing with $r(n), w(m)$, $0 \leq m \leq n-1$, and $v^{i}(m), 0 \leq m \leq n-1,1 \leq i \leq A$. Then, if, for $n>K \geq 0$, we replace $r(n), w(m)$, $K+1 \leq m \leq n-1$, and $v^{i}(m), \quad K+1 \leq m \leq n-1$, $1 \leq i \leq A$, by, respectively, $r_{\mathrm{ub}}(K), w_{\mathrm{ub}}(K)$, and $v_{\mathrm{ub}}^{i}(K)$, $1 \leq i \leq A$, we obtain an upper bound for $d(n)$. Therefore, for $n>K \geq 0$, assuming $w_{\mathrm{ub}}(K)<1$ :

$$
\begin{aligned}
d(n) & \leq \alpha_{S}\left(\prod_{m=0}^{K} w(m)\right) w_{\mathrm{ub}}(K)^{n-K-1} r_{\mathrm{ub}}(K)+\sum_{i=1}^{A} \alpha_{f_{i}} r_{f_{i}} \\
& +\sum_{m=0}^{K} \alpha_{S}\left(\prod_{l=0}^{m-1} w(l)\right) \sum_{i=1}^{A} v^{i}(m) r_{f_{i}} \\
& +\sum_{m=K+1}^{n-1} \alpha_{S}\left(\prod_{l=0}^{K} w(l)\right) w_{\mathrm{ub}}(K)^{m-K-1} \sum_{i=1}^{A} v_{\mathrm{ub}}^{i}(K) r_{f_{i}} \\
& =\nu(K+1) w_{\mathrm{ub}}(K)^{n-K-1} r_{\mathrm{ub}}(K)+\sum_{i=1}^{A} \alpha_{f_{i}} r_{f_{i}} \\
& +\sum_{m=0}^{K} \nu(m) \sum_{i=1}^{A} v^{i}(m) r_{f_{i}} \\
& +\sum_{m=K+1}^{n-1} \nu(K+1) w_{\mathrm{ub}}(K)^{m-K-1} \sum_{i=1}^{A} v_{\mathrm{ub}}^{i}(K) r_{f_{i}} \\
& =\sum_{i=1}^{A} \alpha_{f_{i}} r_{f_{i}}+\sum_{m=0}^{K} \nu(m) \sum_{i=1}^{A} v^{i}(m) r_{f_{i}} \\
& +\nu(K+1) r_{\mathrm{ub}}(K) w_{\mathrm{ub}}(K)^{n-K-1} \\
& +\nu(K+1)\left(\sum_{i=1}^{A} v_{\mathrm{ub}}^{i}(K) r_{f_{i}}\right) \frac{1-w_{\mathrm{ub}}(K)^{n-K-1}}{1-w_{\mathrm{ub}}(K)}=d_{\mathrm{ub}}^{K}(n) .
\end{aligned}
$$

The result $d(n) \geq d_{\mathrm{lb}}^{K}(n)$ for $n>K \geq 0$ can be proven similarly (note that $w_{\mathrm{lb}}(k)<1$ ). It remains to find the upper bound for $d(n), n>K \geq 0$, for the case $w_{\mathrm{ub}}(K)=1$. The only difference in that case is that the last term of the expression of the upper bound has to be replaced by 


$$
\nu(K+1)\left(\sum_{i=1}^{A} v_{\mathrm{ub}}^{i}(K) r_{f_{i}}\right)(n-K-1) .
$$

Bounds for $\operatorname{ETRR}(t)=\sum_{n=0}^{\infty} d(n) e^{-\Lambda t}(\Lambda t)^{n} / n$ ! can be obtained using:

$$
\operatorname{ETRR}_{\mathrm{lb}}^{K}=\sum_{n=0}^{K} d(n) e^{-\Lambda t} \frac{(\Lambda t)^{n}}{n !}+\sum_{n=K+1}^{\infty} d_{\mathrm{lb}}^{K}(n) e^{-\Lambda t} \frac{(\Lambda t)^{n}}{n !}
$$

and

$$
\operatorname{ETRR}_{\mathrm{ub}}^{K}=\sum_{n=0}^{K} d(n) e^{-\Lambda t} \frac{(\Lambda t)^{n}}{n !}+\sum_{n=K+1}^{\infty} d_{\mathrm{ub}}^{K}(n) e^{-\Lambda t} \frac{(\Lambda t)^{n}}{n !}
$$

and the bounds for $d(n), n>K \geq 0$, given by Proposition 2 . The result is given by the following theorem.

Theorem 4. For $K \geq 0$,

$$
\operatorname{ETRR}_{\mathrm{lb}}^{K}(t) \leq \operatorname{ETRR}(t) \leq \operatorname{ETRR}_{\mathrm{ub}}^{K}(t),
$$

where

$$
\begin{aligned}
\operatorname{ETRR}_{\mathrm{lb}}^{K}(t) & =\sum_{n=0}^{K} d(n) e^{-\Lambda t} \frac{(\Lambda t)^{n}}{n !}+B(K) \sum_{n=K+1}^{\infty} e^{-\Lambda t} \frac{(\Lambda t)^{n}}{n !} \\
& +C_{\mathrm{lb}}(K) e^{-\left(1-w_{\mathrm{lb}}(K)\right) \Lambda t} \sum_{n=K+1}^{\infty} e^{-w_{\mathrm{lb}}(K) \Lambda t} \frac{\left(w_{\mathrm{lb}}(K) \Lambda t\right)^{n}}{n !} \\
& +D_{\mathrm{lb}}(K) \sum_{n=K+2}^{\infty}\left(1-w_{\mathrm{lb}}(K)^{n-K-1}\right) e^{-\Lambda t} \frac{(\Lambda t)^{n}}{n !}
\end{aligned}
$$$$
\operatorname{ETRR}_{\mathrm{ub}}^{K}(t)=\sum_{n=0}^{K} d(n) e^{-\Lambda t} \frac{(\Lambda t)^{n}}{n !}+B(K) \sum_{n=K+1}^{\infty} e^{-\Lambda t} \frac{(\Lambda t)^{n}}{n !}
$$$$
+C_{\mathrm{ub}}(K) e^{-\left(1-w_{\mathrm{ub}}(K)\right) \Lambda t} \sum_{n=K+1}^{\infty} e^{-w_{\mathrm{ub}}(K) \Lambda t} \frac{\left(w_{\mathrm{ub}}(K) \Lambda t\right)^{n}}{n !}
$$$$
+I_{w_{\mathrm{ub}}(K)<1} D_{\mathrm{ub}}(K) \sum_{n=K+2}^{\infty}\left(1-w_{\mathrm{ub}}(K)^{n-K-1}\right) e^{-\Lambda t} \frac{(\Lambda t)^{n}}{n !}
$$$$
+I_{w_{\mathrm{ub}}(K)=1} E_{\mathrm{ub}}(K) \sum_{n=K+2}^{\infty}(n-K-1) e^{-\Lambda t} \frac{(\Lambda t)^{n}}{n !},
$$$$
d(n)=\nu(n) r(n)+\sum_{i=1}^{A} \alpha_{f_{i}} r_{f_{i}}+\sum_{m=0}^{n-1} \nu(m) \sum_{i=1}^{A} v^{i}(m) r_{f_{i}},
$$$$
B(K)=\sum_{i=1}^{A} \alpha_{f_{i}} r_{f_{i}}+\sum_{m=0}^{K} \nu(m) \sum_{i=1}^{A} v^{i}(m) r_{f_{i}},
$$$$
C_{\mathrm{lb}}(K)=\frac{\nu(K+1) r_{\mathrm{lb}}(K)}{w_{\mathrm{lb}}(K)^{K+1}}
$$$$
C_{\mathrm{ub}}(K)=\frac{\nu(K+1) r_{\mathrm{ub}}(K)}{w_{\mathrm{ub}}(K)^{K+1}},
$$$$
D_{\mathrm{lb}}(K)=\nu(K+1) \frac{\sum_{i=1}^{A} v_{\mathrm{lb}}^{i}(K) r_{f_{i}}}{1-w_{\mathrm{lb}}(K)},
$$

$$
\begin{gathered}
D_{\mathrm{ub}}(K)=\nu(K+1) \frac{\sum_{i=1}^{A} v_{\mathrm{ub}}^{i}(K) r_{f_{i}}}{1-w_{\mathrm{ub}}(K)}, \\
E_{\mathrm{ub}}(K)=\nu(K+1) \sum_{i=1}^{A} v_{\mathrm{ub}}^{i}(K) r_{f_{i}},
\end{gathered}
$$$$
\nu(n)=\alpha_{S} \prod_{m=0}^{n-1} w(m)
$$

and $r_{\mathrm{lb}}(K), r_{\mathrm{ub}}(K), w_{\mathrm{lb}}(K), w_{\mathrm{ub}}(K)$, and $v_{\mathrm{lb}}^{i}(K), v_{\mathrm{ub}}^{i}(K)$,

$1 \leq i \leq A$, are given by (11)-(13).

Proof. See the Supplement (which can be found on the Computer Society Digital Library at http://computer. org/tc/archives.htm).

Bounds for $\operatorname{EARR}(t)=(1 / t) \int_{0}^{t} \operatorname{ETRR}(\tau) d \tau$ can be obtained using

$$
\operatorname{EARR}_{\mathrm{lb}}^{K}(t)=\frac{1}{t} \int_{0}^{t} \operatorname{ETRR}_{\mathrm{lb}}^{K}(\tau) d \tau
$$

and

$$
\operatorname{EARR}_{\mathrm{ub}}^{K}(t)=\frac{1}{t} \int_{0}^{t} \operatorname{ETRR}_{\mathrm{ub}}^{K}(\tau) d \tau
$$

and the bounds for $\operatorname{ETRR}(t)$ given by Theorem 4. The result is given by the following theorem.

Theorem 5. For $K \geq 0$,

$$
\operatorname{EARR}_{\mathrm{lb}}^{K}(t) \leq E A R R(t) \leq E A R R_{\mathrm{ub}}^{K}(t),
$$

where

$$
\begin{aligned}
& \operatorname{EARR}_{\mathrm{lb}}^{K}(t)=\frac{1}{\Lambda t} \sum_{n=1}^{K+1}\left(\sum_{m=0}^{n-1} d(m)\right) e^{-\Lambda t} \frac{(\Lambda t)^{n}}{n !} \\
& +\frac{1}{\Lambda t}\left(\sum_{n=0}^{K} d(n)\right) \sum_{n=K+2}^{\infty} e^{-\Lambda t} \frac{(\Lambda t)^{n}}{n !} \\
& +\frac{B(K)}{\Lambda t} \sum_{n=K+2}^{\infty}(n-K-1) e^{-\Lambda t} \frac{(\Lambda t)^{n}}{n !} \\
& +\frac{C_{\mathrm{lb}}(K)}{\Lambda t} \frac{w_{\mathrm{lb}}(K)^{K+1}}{1-w_{\mathrm{lb}}(K)} \sum_{n=K+2}^{\infty}\left(1-w_{\mathrm{lb}}(K)^{n-K-1}\right) e^{-\Lambda t} \frac{(\Lambda t)^{n}}{n !} \\
& +\frac{D_{\mathrm{lb}}(K)}{\Lambda t} \sum_{n=K+3}^{\infty}\left(\sum_{m=K+2}^{n-1}\left(1-w_{\mathrm{lb}}(K)^{m-K-1}\right)\right) e^{-\Lambda t} \frac{(\Lambda t)^{n}}{n !},
\end{aligned}
$$




$$
\begin{aligned}
& E A R R_{\mathrm{ub}}^{K}(t)=\frac{1}{\Lambda t} \sum_{n=1}^{K+1}\left(\sum_{m=0}^{n-1} d(m)\right) e^{-\Lambda t} \frac{(\Lambda t)^{n}}{n !} \\
& +\frac{1}{\Lambda t}\left(\sum_{n=0}^{K} d(n)\right) \sum_{n=K+2}^{\infty} e^{-\Lambda t} \frac{(\Lambda t)^{n}}{n !} \\
& +\frac{B(K)}{\Lambda t} \sum_{n=K+2}^{\infty}(n-K-1) e^{-\Lambda t} \frac{(\Lambda t)^{n}}{n !} \\
& +I_{w_{\mathrm{ub}}(K)<1} \frac{C_{\mathrm{ub}}(K)}{\Lambda t} \frac{w_{\mathrm{ub}}(K)^{K+1}}{1-w_{\mathrm{ub}}(K)} \sum_{n=K+2}^{\infty}\left(1-w_{\mathrm{ub}}(K)^{n-K-1}\right) \\
& +I_{w_{\mathrm{ub}}(K)=1} \frac{C_{\mathrm{ub}}(K)}{\Lambda t} \sum_{n=K+2}^{\infty}(n-K-1) e^{-\Lambda t} \frac{(\Lambda t)^{n}}{n !} \\
& +I_{w_{\mathrm{ub}}(K)<1} \frac{D_{\mathrm{ub}}(K)}{\Lambda t} \sum_{n=K+3}^{\infty}\left(\sum_{m=K+2}^{n-1}\left(1-w_{\mathrm{ub}}(K)^{m-K-1}\right)\right) \\
& +I_{w_{\mathrm{ub}}(K)=1} \frac{E_{\mathrm{ub}}(K)}{\Lambda t} \sum_{n=K+3}^{\infty} \frac{(\Lambda t)^{n}}{2} \frac{(\Lambda t)^{n}}{n !}
\end{aligned}
$$

$d(n), B(K), C_{\mathrm{lb}}(K), C_{\mathrm{ub}}(K), D_{\mathrm{lb}}(K), D_{\mathrm{ub}}(K)$, and $E_{\mathrm{ub}}(K)$ being those defined in Theorem 4.

Proof. See the Supplement (which can be found on the Computer Society Digital Library at http://computer. org/tc/archives.htm).

The randomization with quasistationarity detection method uses the bounds for $\operatorname{ETRR}(t)$ and $\operatorname{EARR}(t)$ given by, respectively, Theorems 4 and 5. Combining (3)-(5) with (7)-(10), it follows that $r(n), w(n), v^{i}(n), 1 \leq i \leq A$, $0 \leq n \leq K$, can be computed from the vectors $\mathbf{o}(n), \mathbf{r}(n)$, $\mathbf{w}(n), \mathbf{v}^{i}(n), 1 \leq i \leq A, 0 \leq n \leq K$, using:

$$
\begin{aligned}
r(n) & =\frac{\boldsymbol{\alpha}_{S}^{T} \mathbf{r}(n)}{\boldsymbol{\alpha}_{S}^{T} \mathbf{o}(n)}, \\
w(n) & =\frac{\boldsymbol{\alpha}_{S}^{T} \mathbf{w}(n)}{\boldsymbol{\alpha}_{S}^{T} \mathbf{o}(n)}, \\
v^{i}(n) & =\frac{\boldsymbol{\alpha}_{S}^{T} \mathbf{v}^{i}(n)}{\boldsymbol{\alpha}_{S}^{T} \mathbf{o}(n)}, \quad 1 \leq i \leq A ;
\end{aligned}
$$

$r_{\mathrm{lb}}(K), r_{\mathrm{ub}}(K), w_{\mathrm{lb}}(K), w_{\mathrm{ub}}(K), v_{\mathrm{lb}}^{i}(K), v_{\mathrm{ub}}^{i}(K), 1 \leq i \leq A$, can be computed from the vectors $\mathbf{o}(K), \mathbf{r}(K), \mathbf{w}(K), \mathbf{v}^{i}(K)$, $1 \leq i \leq A$, using (11)-(13); finally, the vectors $\mathbf{o}(n), \mathbf{r}(n)$, $\mathbf{w}(n), \mathbf{v}^{i}(n), 1 \leq i \leq A, 0 \leq n \leq K$, can be computed recursively making matrix-vector multiplications, e.g., $\mathbf{o}(n), 0 \leq n \leq K$, can be computed using $\mathbf{o}(0)=1$ and $\mathbf{o}(k+1)=\mathbf{P}_{S, S} \mathbf{o}(k), 0 \leq k \leq K-1$. The following theorem ensures that, by choosing a large enough $K$, the method will be able to compute the measure with arbitrary accuracy, ignoring, of course, roundoff errors. Consider, for instance, the $\operatorname{ETRR}(t)$ measure. Then, $\varepsilon$ being an error control parameter, $K$ can be selected using

$$
K=\min \left\{m \geq 0: \operatorname{ETRR}_{\mathrm{ub}}^{m}(t)-\operatorname{ETRR}_{\mathrm{lb}}^{m}(t) \leq 2 \varepsilon\right\},
$$

and $\operatorname{ETRR}(t)$ can be estimated with absolute error $\leq \varepsilon$ by $\left(\operatorname{ETRR}_{\mathrm{lb}}^{K}(t)+\operatorname{ETRR}_{\mathrm{ub}}^{K}(t)\right) / 2$.
Theorem 6. The bounds $\operatorname{ETRR}_{\mathrm{lb}}^{K}(t)$ and $E T R R_{\mathrm{ub}}^{K}(t)$ given by Theorem 4 become monotonically arbitrarily tight as $K \rightarrow \infty$ and the bounds $\operatorname{EARR}_{\mathrm{lb}}^{K}(t)$ and $\operatorname{EARR}_{\mathrm{ub}}^{K}(t)$ given by Theorem 5 become monotonically arbitrarily tight as $K \rightarrow \infty$.

Proof. From Theorem 3, part 2, $r_{\mathrm{lb}}(K), w_{\mathrm{lb}}(K)$ and $v_{\mathrm{lb}}^{i}(K)$, $1 \leq i \leq A$, increase monotonically with $K$ and $r_{\mathrm{ub}}(K)$, $w_{\mathrm{ub}}(K)$, and $v_{\mathrm{ub}}^{i}(K), 1 \leq i \leq A$, decrease monotonically with $K$. For $n>K$, we can write (see the proof of Proposition 2):

$$
\begin{aligned}
d_{\mathrm{ub}}^{K}(n) & =\alpha_{S}\left(\prod_{m=0}^{K} w(m)\right) w_{\mathrm{ub}}(K)^{n-K-1} r_{\mathrm{ub}}(K)+\sum_{i=1}^{A} \alpha_{f_{i}} r_{f_{i}} \\
& +\sum_{m=0}^{K} \alpha_{S}\left(\prod_{l=0}^{m-1} w(l)\right) \sum_{i=1}^{A} v^{i}(m) r_{f_{i}} \\
& +\sum_{m=K+1}^{n-1} \alpha_{S}\left(\prod_{l=0}^{K} w(l)\right) w_{\mathrm{ub}}(K)^{m-K-1} \sum_{i=1}^{A} v_{\mathrm{ub}}^{i}(K) r_{f_{i}} .
\end{aligned}
$$

Similarly, for $n>K+1$,

$$
\begin{aligned}
& d_{\mathrm{ub}}^{K+1}(n)=\alpha_{S}\left(\prod_{m=0}^{K+1} w(m)\right) w_{\mathrm{ub}}(K+1)^{n-K-2} r_{\mathrm{ub}}(K+1) \\
& +\sum_{i=1}^{A} \alpha_{f_{i}} r_{f_{i}}+\sum_{m=0}^{K+1} \alpha_{S}\left(\prod_{l=0}^{m-1} w(l)\right) \sum_{i=1}^{A} v^{i}(m) r_{f_{i}} \\
& +\sum_{m=K+2}^{n-1} \alpha_{S}\left(\prod_{l=0}^{K+1} w(l)\right) w_{\mathrm{ub}}(K+1)^{m-K-2} \sum_{i=1}^{A} v_{\mathrm{ub}}^{i}(K+1) r_{f_{i}} \\
& =\alpha_{S}\left(\prod_{m=0}^{K+1} w(m)\right) w_{\mathrm{ub}}(K+1)^{n-K-2} r_{\mathrm{ub}}(K+1)+\sum_{i=1}^{A} \alpha_{f_{i}} r_{f_{i}} \\
& +\sum_{m=0}^{K} \alpha_{S}\left(\prod_{l=0}^{m-1} w(l)\right) \sum_{i=1}^{A} v^{i}(m) r_{f_{i}} \\
& +\alpha_{S}\left(\prod_{l=0}^{K} w(l)\right) \sum_{i=1}^{A} v^{i}(K+1) r_{f_{i}} \\
& +\sum_{m=K+2}^{n-1} \alpha_{S}\left(\prod_{l=0}^{K+1} w(l)\right) w_{\mathrm{ub}}(K+1)^{m-K-2} \sum_{i=1}^{A} v_{\mathrm{ub}}^{i}(K+1) r_{f_{i}} .
\end{aligned}
$$

For $n>K+1, w(K+1) \leq w_{\mathrm{ub}}(K)$,

$$
w_{\mathrm{ub}}(K+1) \leq w_{\mathrm{ub}}(K),
$$

and $r_{\mathrm{ub}}(K+1) \leq r_{\mathrm{ub}}(K)$ imply

$$
\begin{aligned}
& \alpha_{S}\left(\prod_{m=0}^{K+1} w(m)\right) w_{\mathrm{ub}}(K+1)^{n-K-2} r_{\mathrm{ub}}(K+1) \\
& \leq \alpha_{S}\left(\prod_{m=0}^{K} w(m)\right) w_{\mathrm{ub}}(K)^{n-K-1} r_{\mathrm{ub}}(K)
\end{aligned}
$$

and $w(K+1) \leq w_{\mathrm{ub}}(K), w_{\mathrm{ub}}(K+1) \leq w_{\mathrm{ub}}(K)$,

$$
v^{i}(K+1) \leq v_{\mathrm{ub}}^{i}(K),
$$

$1 \leq i \leq A$, and $v_{\mathrm{ub}}^{i}(K+1) \leq v_{\mathrm{ub}}^{i}(K), 1 \leq i \leq A$, imply 


$$
\begin{aligned}
& \alpha_{S}\left(\prod_{l=0}^{K} w(l)\right) \sum_{i=1}^{A} v^{i}(K+1) r_{f_{i}} \\
& +\sum_{m=K+2}^{n-1} \alpha_{S}\left(\prod_{l=0}^{K+1} w(l)\right) w_{\mathrm{ub}}(K+1)^{m-K-2} \sum_{i=1}^{A} v_{\mathrm{ub}}^{i}(K+1) r_{f_{i}} \\
& \leq \sum_{m=K+1}^{n-1} \alpha_{S}\left(\prod_{l=0}^{K} w(l)\right) w_{\mathrm{ub}}(K)^{m-K-1} \sum_{i=1}^{A} v_{\mathrm{ub}}^{i}(K) r_{f_{i}},
\end{aligned}
$$

and both inequalities imply, for $n>K+1$,

$$
d_{\mathrm{ub}}^{K+1}(n) \leq d_{\mathrm{ub}}^{K}(n) .
$$

Then, for $n>K, d_{\mathrm{ub}}^{K}(n)$ decreases monotonically with $K$. That, for $n>K, d_{\mathrm{lb}}^{K}(n)$ increases monotonically with $K$ can be proven similarly.

Since

$\operatorname{ETRR}_{\mathrm{ub}}^{K}(t)=\sum_{n=0}^{K} d(n) e^{-\Lambda t} \frac{(\Lambda t)^{n}}{n !}+\sum_{n=K+1}^{\infty} d_{\mathrm{ub}}^{K}(n) e^{-\Lambda t} \frac{(\Lambda t)^{n}}{n !}$,

$$
\begin{aligned}
& \operatorname{ETRR}_{\mathrm{ub}}^{K}(t)-\operatorname{ETRR}_{\mathrm{ub}}^{K+1}(t) \\
& =\left(d_{\mathrm{ub}}^{K}(K+1)-d(K+1)\right) e^{-\Lambda t} \frac{(\Lambda t)^{K+1}}{(K+1) !} \\
& +\sum_{n=K+2}^{\infty}\left(d_{\mathrm{ub}}^{K}(n)-d_{\mathrm{ub}}^{K+1}(n)\right) e^{-\Lambda t} \frac{(\Lambda t)^{n}}{n !},
\end{aligned}
$$

which, together with $d_{\mathrm{ub}}^{K}(K+1) \geq d(K+1)$ and $d_{\mathrm{ub}}^{K+1}(n) \leq d_{\mathrm{ub}}^{K}(n)$, implies that $E T R R_{\mathrm{ub}}^{K}(t)$ decreases monotonically with $K$. That $E T R R_{\mathrm{lb}}^{K}(t)$ increases monotonically with $K$ can be proven similarly. Using Theorem 3 , part 1, $\lim _{K \rightarrow \infty} w_{\mathrm{lb}}(K)=\lim _{K \rightarrow \infty} w_{\mathrm{ub}}(K)=\mathbf{u}^{T} \mathbf{w}$, $\mathbf{u}$ being the Perron-Frobenius left eigenvector of $\mathbf{P}_{S, S}$, which has all its components strictly positive. But, since the states in $S$ are transient in $\widehat{X}, P_{j, S}<1$ for at least some $j \in S$ and, then, $\mathbf{u}^{T} \mathbf{w}<\mathbf{u}^{T} \mathbf{1}=1$. This implies that, for large enough $K$, $w_{\mathrm{lb}}(K), w_{\mathrm{ub}}(K)$ will be $<1$. Using, then, the expressions for $d_{\mathrm{lb}}^{K}(n)$ and $d_{\mathrm{ub}}^{K}(n)$ given by Proposition 2, for large enough $K, d_{\mathrm{lb}}^{K}(n)$ and $d_{\mathrm{ub}}^{K}(n)$ will be uniformly bounded for $n>K$. Using $\operatorname{ETRR}(t)=\sum_{n=0}^{\infty} d(n) e^{-\Lambda t}(\Lambda t)^{n} / n$ ! and (14) together with $\lim _{K \rightarrow \infty} \sum_{n=K+1}^{\infty} e^{-\Lambda t}(\Lambda t)^{n} / n !=0$ and that $d_{\mathrm{ub}}^{K}(n)$ is, for large enough $K$, uniformly bounded, it is easy to prove that $\lim _{K \rightarrow \infty} E T R R_{\mathrm{ub}}^{K}(t)=E T R R(t)$. That $\lim _{K \rightarrow \infty} E T R R_{\mathrm{lb}}^{K}(t)=E T R R(t)$ can be proven similarly, completing the proof that $\operatorname{ETRR}_{\mathrm{lb}}^{K}(t)$ and $\operatorname{ETRR}_{\mathrm{ub}}^{K}(t)$ become monotonically arbitrarily tight as $K \rightarrow \infty$.

That $E A R R_{\mathrm{ub}}^{K}(t)$ decreases monotonically with $K$ follows from $E A R R_{\mathrm{ub}}^{K}(t)=\left(\int_{0}^{t} E T R R_{\mathrm{ub}}^{K}(\tau) d \tau\right) / t$ and the fact that $E T R R_{\mathrm{ub}}^{K}(t)$ decreases monotonically with $K$. That $E A R R_{\mathrm{lb}}^{K}(t)$ increases monotonically with $K$ can be proven similarly. From (14) and $E A R R_{\mathrm{ub}}^{K}(t)=\left(\int_{0}^{t} E T R R_{\mathrm{ub}}^{K}(\tau) d \tau\right) / t$, we obtain

$$
\begin{aligned}
\operatorname{EARR}_{\mathrm{ub}}^{K}(t) & =\sum_{n=0}^{K} d(n) \frac{1}{t} \int_{0}^{t} e^{-\Lambda \tau} \frac{(\Lambda \tau)^{n}}{n !} d \tau \\
& +\sum_{n=K+1}^{\infty} d_{\mathrm{ub}}^{K}(n) \frac{1}{t} \int_{0}^{t} e^{-\Lambda \tau} \frac{(\Lambda \tau)^{n}}{n !} d \tau .
\end{aligned}
$$

Using, then,

$$
\begin{aligned}
\operatorname{EARR}(t) & =\left(\int_{0}^{t} \operatorname{ETRR}(\tau) d \tau\right) / t \\
& =\sum_{n=0}^{\infty} d(n)\left(\int_{0}^{t} e^{-\Lambda \tau}(\Lambda \tau)^{n} / n !\right) / t
\end{aligned}
$$

together with

$$
\begin{aligned}
& \lim _{K \rightarrow \infty} \sum_{n=K+1}^{\infty}\left(\int_{0}^{t} e^{-\Lambda \tau}(\Lambda \tau)^{n} / n ! d \tau\right) / t \\
& =\lim _{K \rightarrow \infty}(1 / \Lambda) \sum_{n=K+1}^{\infty} \sum_{m=n+1}^{\infty} e^{-\Lambda t}(\Lambda t)^{m} / m ! \\
& =\lim _{K \rightarrow \infty}(1 / \Lambda) \sum_{n=K+2}^{\infty}(n-K-1) e^{-\Lambda t}(\Lambda t)^{n} / n !=0
\end{aligned}
$$

and that $d_{\mathrm{ub}}^{K}(n)$ is, for large enough $K$, uniformly bounded, it is easy to prove that

$$
\lim _{K \rightarrow \infty} E A R R_{\mathrm{ub}}^{K}(t)=\operatorname{EARR}(t) .
$$

That $\lim _{K \rightarrow \infty} E A R R_{\mathrm{lb}}^{K}(t)=E A R R(t)$ can be proven similarly, completing the proof that $E A R R_{\mathrm{lb}}^{K}(t)$ and $E A R R_{\mathrm{ub}}^{K}(t)$ become monotonically arbitrarily tight as $K \rightarrow \infty$.

Computing the factors $1-w_{\mathrm{lb}}(K), 1-w_{\mathrm{ub}}(K)$, $1-w_{\mathrm{lb}}(K)^{n}$, and $1-w_{\mathrm{ub}}(K)^{n}, n>1$ involved in the expressions for the bounds for $\operatorname{ETRR}(t)$ and $\operatorname{EARR}(t)$ given by, respectively, Theorems 4 and 5 in the trivial way will be numerically unstable when $w_{\mathrm{lb}}(K)$ and $w_{\mathrm{ub}}(K)$ are very close to 1 . The problem can be solved as follows: From $\mathbf{w}+\sum_{i=1}^{A} \mathbf{v}^{i}=\left(P_{j, S}+\sum_{i=1}^{A} P_{j, f_{i}}\right)_{j \in S}=\mathbf{1}$,

$$
\mathbf{P}_{S, S}^{n} \mathbf{w}+\sum_{i=1}^{A} \mathbf{P}_{S, S}^{n} \mathbf{v}^{i}=\mathbf{P}_{S, S}^{n} \mathbf{1}
$$

and (7), (9), (10)

$$
\mathbf{w}(n)+\sum_{i=1}^{A} \mathbf{v}^{i}(n)=\mathbf{o}(n) .
$$

Using (15), the following numerically stable expressions for $1-w_{\mathrm{lb}}(k)$ and $1-w_{\mathrm{ub}}(K)$ can be obtained:

$$
\begin{aligned}
1-w_{\mathrm{lb}}(K) & =1-\min _{j \in S} \frac{w_{j}(K)}{o_{j}(K)}=\max _{j \in S} \frac{o_{j}(K)-w_{j}(K)}{o_{j}(K)} \\
& =\max _{j \in S} \frac{\sum_{i=1}^{A} v_{j}^{i}(K)}{o_{j}(K)}, \\
1-w_{\mathrm{ub}}(K) & =1-\max _{j \in S} \frac{w_{j}(K)}{o_{j}(K)}=\min _{j \in S} \frac{o_{j}(K)-w_{j}(K)}{o_{j}(K)} \\
& =\min _{j \in S} \frac{\sum_{i=1}^{A} v_{j}^{i}(K)}{o_{j}(K)} .
\end{aligned}
$$

The factors $1-w_{\mathrm{lb}}(K)^{n}$ and $1-w_{\mathrm{ub}}(K)^{n}, n>1$, can then be computed with numerical stability from, respectively, $w_{\mathrm{lb}}(K), 1-w_{\mathrm{lb}}(K)$ and $w_{\mathrm{ub}}(K), 1-w_{\mathrm{ub}}(K)$ using the following proposition and $1-w_{\mathrm{ub}}(K)^{n}=0$ for $1-w_{\mathrm{ub}}(K)=0$.

Proposition 3. Let $f(x)=1-x^{n}, n>1$. Then, assuming that both $x$ and $1-x$ are known with high accuracy, 
$f(x), 0<x<1$ can be computed with high accuracy in the following way:

1. for $n(1-x)<0.1$, using as many terms as required of the alternating decreasing series

$$
f(x)=\sum_{k=1}^{n}(-1)^{k+1}\left(\begin{array}{l}
n \\
k
\end{array}\right)(1-x)^{k}
$$

2. for $n(1-x) \geq 0.1$, using $f(x)=1-x^{n}$.

Proof. See the Supplement (which can be found on the Computer Society Digital Library at http://computer. org/tc/archives.htm).

Vectors $\mathbf{o}(n)$ and $\mathbf{w}(n)$ are related. Using $\mathbf{P}_{S, S} \mathbf{1}=\left(P_{j, S}\right)_{j \in S}=\mathbf{w}$, for $n \geq 0$, we obtain (7), (9):

$$
\mathbf{o}(n+1)=\mathbf{P}_{S, S}^{n+1} \mathbf{1}=\mathbf{P}_{S, S}^{n} \mathbf{P}_{S, S} \mathbf{1}=\mathbf{P}_{S, S}^{n} \mathbf{w}=\mathbf{w}(n) .
$$

Equation (18) can be used to avoid the computation of $\mathbf{o}(n)$, $1 \leq n \leq K$.

Efficient and stable computation of the infinite summatories of the form $\sum_{n=M}^{\infty} \omega(n) e^{-\lambda} \lambda^{n} / n$ ! involved in the expressions for the bounds for $\operatorname{ETRR}(t)$ and $\operatorname{EARR}(t)$ given by, respectively, Theorems 4 and 5 is a delicate issue. The method uses the procedures described in the Supplement, with implicit use of the previously described numerically stable ways of computing $1-w_{\mathrm{lb}}(K)$, $1-w_{\mathrm{ub}}(K), \quad 1-w_{\mathrm{lb}}(K)^{n}$, and $1-w_{\mathrm{ub}}(K)^{n}, n>1$. The procedures compute, in a numerically stable way, the summatories with relative error $<\delta, \delta$ being the "epsilon" constant of the computer (smallest floating-point number $x>0$ such that $1+x$ yields a result different from 1 ) and exploit the fact that, for large $\lambda$ and $M<\lambda$, only the terms $\omega(n) e^{-\lambda} \lambda^{n} / n$ ! within a few $\sqrt{\lambda}$ apart from $\lambda$ can have significant contributions to the summatory. However, even using those procedures, computation of the expressions which give the bounds for $\operatorname{ETRR}(t)$ and $\operatorname{EARR}(t)$ for $K=$ $0,1,2, \ldots$ until they become tight enough introduces an overhead which can be relatively substantial when the model is not very large. To reduce that overhead, the method uses a lookahead approach based on the assumption that, for large $K$, the band $b(K)$ defined by the bounds is $\approx A \rho^{K}, A>0,0<\rho<1 .^{2}$ With the " $K$ " parameter equal to $K+1$, the attenuation parameter $\rho$ can be estimated from $b(K)$ and $b(K+1)$ by $\rho_{K+1}=b(K+1) / b(K)$. From $\rho_{K+1}$ and $b(K+1)$ the value by which the " $K$ " parameter will have to be increased beyond $K+1$ to have an error $\leq \varepsilon$ can be estimated by

$$
\Delta K_{K+1}=\frac{\log (2 \varepsilon)-\log (b(K+1))}{\log \left(\rho_{K+1}\right)} .
$$

The same estimation can be made for the " $K$ " parameter one unit greater, yielding an estimate for the value by which the " $K$ " parameter will have to be increased beyond $K+2$

2. We have not been able to prove that $\lim _{K \rightarrow \infty} b(K) /\left(A \rho^{K}\right)=1$ for some $A>0$ and some $\rho, 0<\rho<1$. However, the lookahead approach based on that assumption has worked well in practice in all examples we have tried, i.e., the $K$ obtained using the technique is the one strictly required.

$$
\Delta K_{K+2}=\frac{\log (2 \varepsilon)-\log (b(K+2))}{\log \left(\rho_{K+2}\right)} .
$$

The lookahead approach consists of, if $\Delta K_{K+1}-1$ and $\Delta K_{K+2}$ are sufficiently close, increasing the " $K$ " parameter beyond $K+2$ up to $K^{\prime}=K+2+\min \left\{\left\lfloor\Delta K_{K+2} / 2\right\rfloor, 100\right\}$ before computing the bounds for the measure again, where the constant 100 limits the extent of the lookahead.

To clarify, Fig. 2 gives a C-like algorithmic description of the randomization with quasistationarity detection method for the measure $\operatorname{ETRR}(t)$. The algorithmic description has, as inputs, the CTMC $X$, the subset of transient states $S$, the number of absorbing states $A$, the absorbing states $f_{i}$, $1 \leq i \leq A$, the reward rate structure $r_{i}, i \in \Omega$, the initial probability distribution vector $\alpha=\left(\alpha_{i}\right)_{i \in \Omega}$, the error control parameter $\varepsilon$, the number of time points $n$ at which the measure has to be computed, and the time points $t_{1}, t_{2}, \ldots, t_{n}>0$, and, as outputs, estimates $\operatorname{ETRR}\left(t_{i}\right), 1 \leq$ $i \leq n$ for the measure at the required time points $t_{i}, 1 \leq i \leq$ $n$ with absolute error $\leq \varepsilon$. It is assumed that the rewarded CTMC has the required properties, which are easy to check. In the description, $\mathbf{1}$ denotes an $|S|$-vector with all its components equal to 1 . The description makes explicit the use of (18) to avoid the computation of the vectors $\mathbf{o}(n)$, $1 \leq n \leq K$, and the numerically stable computation of $1-$ $w_{\mathrm{lb}}(K)$ and $1-w_{\mathrm{ub}}(K)$, called, respectively, $w_{\mathrm{lb}}(K)^{c}$ and $w_{\mathrm{ub}}(K)^{c}$, using (16) and (17). The particular case $r_{i}=0, i \in$ $S$ receives a special treatment by exploiting the fact that, in that case, $r(n)=0,0 \leq n \leq K$ and $r_{\mathrm{lb}}(K)=r_{\mathrm{ub}}(K)=0$. The randomization with quasistationarity detection method for the measure $\operatorname{EARR}(t)$ can be described similarly. The method has, for both measures, the same excellent numerical stability as the standard randomization method.

\section{Analysis}

In this section, we will analyze the performance of randomization with quasistationarity detection and will compare it with those of standard randomization and regenerative randomization. We will consider a class $\mathrm{C}^{\prime}$ model and a model outside class $C^{\prime}$ for which there is no natural selection for the regenerative state and regenerative randomization for a reasonable selection for the regenerative state performs poorly. All CPU times are measured in a workstation with a Sun-Blade-1000 processor and 4 GB of memory.

\subsection{Class C' model}

The model is a parametric unreliability model of a faulttolerant storage system made up of $N_{\mathrm{SS}}$ 5-level RAID subsystems, each one comprised of eight disks, two redundant disk controllers, and two redundant power supplies. The power supplies work in cold standby redundancy. The system is operational if all RAID subsystems are up. The availability of an unlimited number of repairmen to repair failed components is assumed. The failure rate of a disk in an up RAID subsystem not under reconstruction is $2 \times 10^{-6} \mathrm{~h}^{-1}$, the failure rate of a disk in an up RAID subsystem with one disk under reconstruction is $3 \times 10^{-6} \mathrm{~h}^{-1}$, the failure rate of a controller in an up RAID subsystem with two unfailed controllers is $5 \times 10^{-6} \mathrm{~h}^{-1}$, the failure rate of a controller in an up RAID subsystem with 


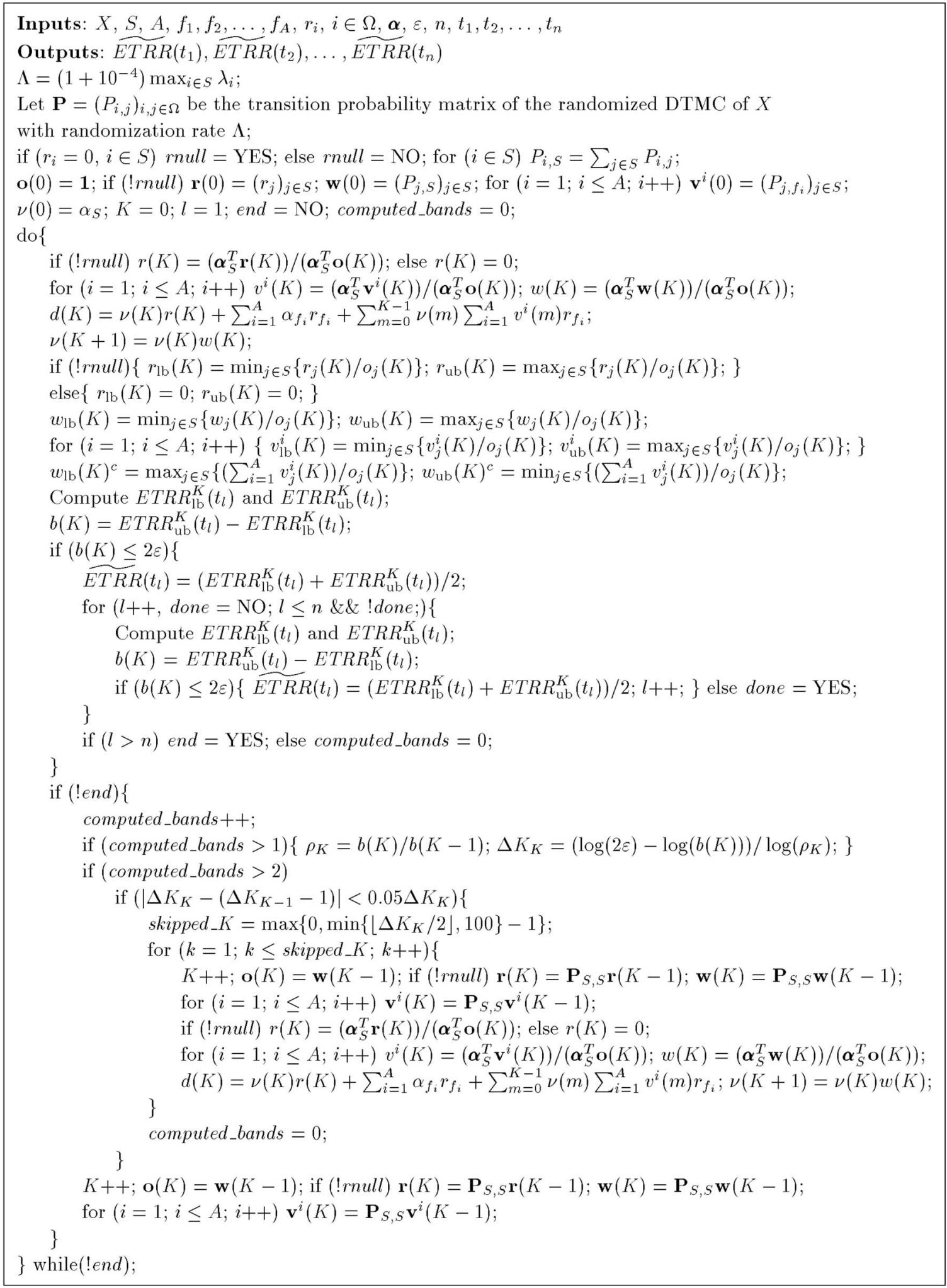

Fig. 2. Algorithmic description of the randomization with quasistationarity detection method for the $\operatorname{ETRR}(t)$ measure.

only one unfailed controller is $8 \times 10^{-6} \mathrm{~h}^{-1}$, the failure rate of the active power supply in an up RAID subsystem is $6 \times 10^{-6} \mathrm{~h}^{-1}$, the coverage to controller failures is 0.99 , the coverage to power supply failures is 0.995 , the disk reconstruction rate is $0.4 \mathrm{~h}^{-1}$, and the repair rate of failed components in up RAID subsystems is $0.125 \mathrm{~h}^{-1}$. The
CTMC $X$ has a state space $S \cup\left\{f_{1}\right\}$, where $S$ includes the operational states and entry into the absorbing state $f_{1}$ models system failure. The generic $\operatorname{ETRR}(t)$ measure considered in this paper with $r_{i}=0, i \in S$ and $r_{f_{1}}=1$ gives the unreliability of the fault-tolerant storage system at time $t, \operatorname{ur}(t)$. To reduce its size, the model is generated from a 


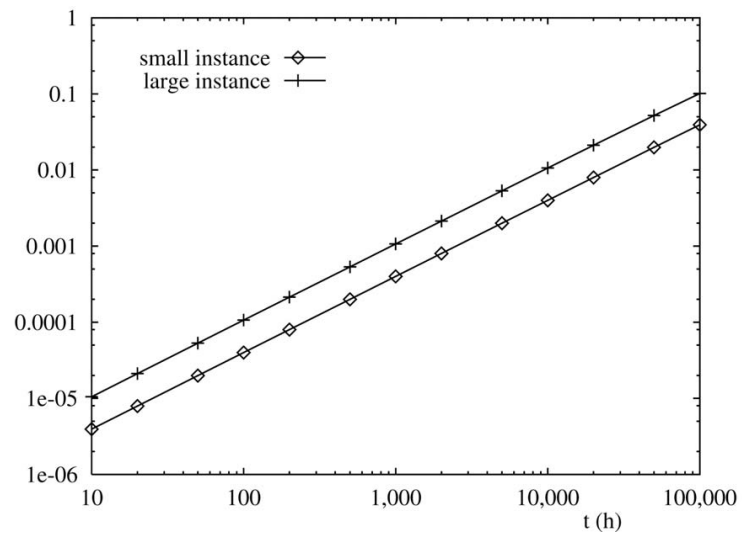

Fig. 3. Unreliability as a function of $t$ for both instances of the example in class C'.

high-level description which exploits the fact that the RAID subsystems are identical. A more detailed description of the model can be found in [7], where the $N_{\mathrm{SS}}$ parameter is called $N$. It is assumed that, initially, the system is in the state in which all RAID subsystems are in their fully operational state, i.e., without any failed component and with no disk under reconstruction.

The model belongs to class $C^{\prime}$, the state $o$ being the state in which all RAID subsystems are in their fully operational state, and that state is the natural selection for the regenerative state in the regenerative randomization method. The performance of regenerative randomization relative to standard randomization is affected by the size of the model. This is because, in the second phase of that method, a truncated transformed model, with almost the same maximum output rate as the original model and with a size which is mainly determined by the ratio $R$ between the maximum and the minimum output rates from states in $S-\{o\}$, has to be solved by standard randomization and the relative cost of that phase depends on the size of the original model. To take that into account, we consider two instances of the parametric model. The first instance is

TABLE 1

Value of the Truncation Parameter $K$ in Randomization with Quasistationarity Detection (RQD) and

Regenerative Randomization (RR) and Value of the Truncation Parameter $N$ in Standard Randomization (SR) for Both Instances of the Model in Class C'

\begin{tabular}{|c|ccc|ccc|}
\hline \hline & \multicolumn{3}{|c|}{ small instance } & \multicolumn{3}{c|}{ large instance } \\
$t(\mathrm{~h})$ & $K(\mathrm{RQD})$ & $K(\mathrm{RR})$ & $N(\mathrm{SR})$ & $K(\mathrm{RQD})$ & $K(\mathrm{RR})$ & $N(\mathrm{SR})$ \\
\hline \hline 10 & 39 & 42 & 54 & 102 & 88 & 104 \\
50 & 124 & 129 & 167 & 309 & 316 & 369 \\
100 & 203 & 212 & 290 & 543 & 557 & 672 \\
500 & 289 & 311 & 1,180 & 825 & 885 & 2,931 \\
1,000 & 302 & 325 & 2,238 & 861 & 923 & 5,666 \\
5,000 & 328 & 351 & 10,385 & 933 & 995 & 27,034 \\
10,000 & 339 & 362 & 20,396 & 963 & 1,025 & 53,460 \\
50,000 & 364 & 387 & 99,499 & 1,029 & 1,092 & 263,266 \\
100,000 & 374 & 397 & 197,827 & 1,015 & 1,120 & 524,624 \\
\hline \hline
\end{tabular}

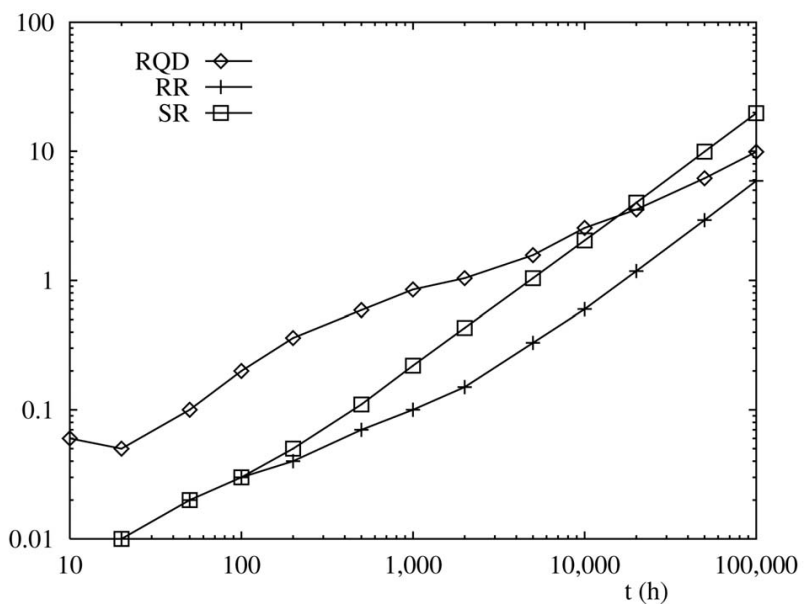

Fig. 4. CPU times consumed by the methods in seconds for the small instance of the example in class C' as a function of $t$.

obtained by making $N_{\mathrm{SS}}=3$ and yields a CTMC with 365 states and 3,484 transitions. The second instance is obtained by making $N_{\mathrm{SS}}=8$ and yields a CTMC with 75,583 states and 1,384,542 transitions. Fig. 3 plots $u r(t)$ for both instances. All methods are run with a single target time $t$ and a required error $\varepsilon=10^{-10}$. For the regenerative randomization method, the state $o$ is taken as regenerative state. Table 1 gives the values of the truncation parameter $K$ in randomization with quasistationarity detection (RQD), the truncation parameter $K$ in regenerative randomization (RR), and the truncation parameter $N$ in standard randomization (SR) for both instances of the model. The CPU times consumed by the methods are given in, respectively, Figs. 4 and 5.

The value of the truncation parameter $K$ in randomization with quasistationarity detection is similar to the value of the truncation parameter $K$ in regenerative randomization and, for large $t$, much smaller than the value of the truncation parameter $N$ in standard randomization, which, for large $t$, increases approximately linearly with $t$. This makes both randomization with quasistationarity detection and regenerative randomization significantly less costly

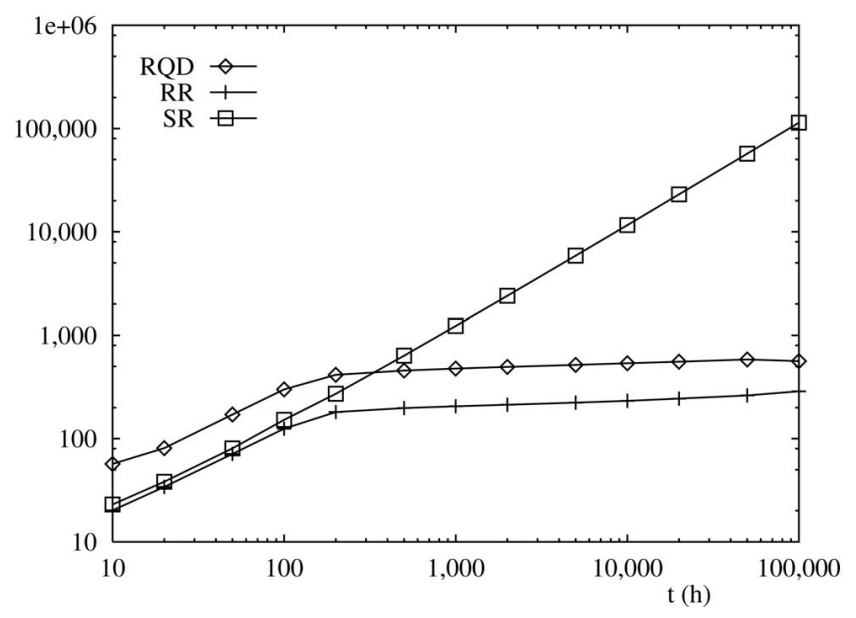

Fig. 5. CPU times consumed by the methods in seconds for the large instance of the example in class $C^{\prime}$ as a function of $t$. 


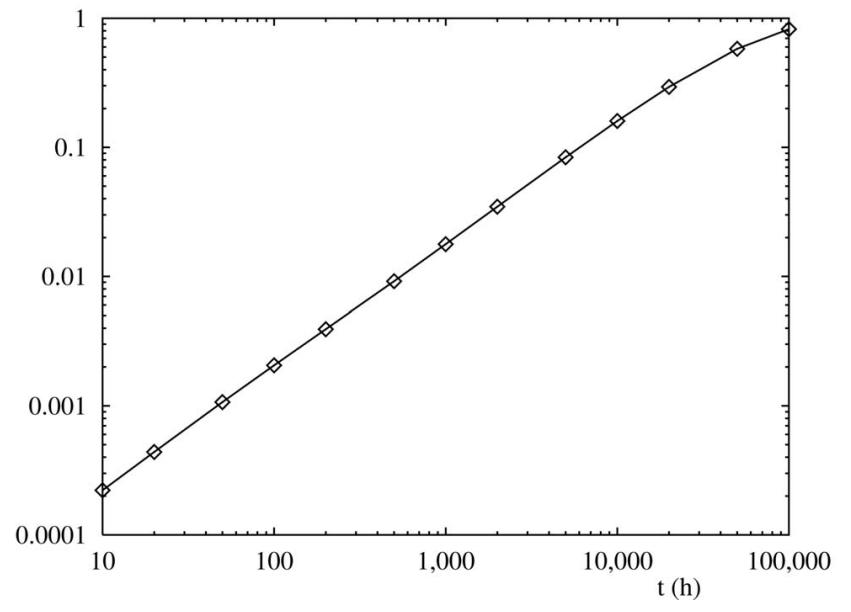

Fig. 6. Unreliability as a function of $t$ for the example outside class C'.

than standard randomization for large models and large $t$. For small models, the cost of computing the infinite summatories in randomization with quasistationarity detection and the cost of solving the truncated transformed model in regenerative randomization are relatively important and the methods are not much faster than standard randomization. Randomization with quasistationarity detection is always more expensive than regenerative randomization. This is because the cost of that method per step (increment of $K$ ) is larger than the cost per step of regenerative randomization. The computational cost of regenerative randomization would be higher when the initial probability distribution of the model is not concentrated in the state $o$, but would not double the cost of the case considered in the numerical experiments (initial probability distribution concentrated in state o) [4], [5]. Furthermore, the example has $A=1$ and null reward rates in $S$ and, then, covers the most favorable case for randomization with quasistationarity detection. Therefore, we can conclude that, for class $C^{\prime}$ models, regenerative randomization seems to outperform randomization with quasistationarity detection.

\subsection{Model Outside Class C'}

The model corresponds to a fault-tolerant multiserver system including 20 servers of type 1 and 20 servers of type 2 . The system is operational if there are at least 16 servers of each type unfailed and there has not been any coverage failure. Servers have hypoexponential lifetime distributions with parameters $q=0.1, \lambda_{\mathrm{F}}=0.01 \mathrm{~h}^{-1}$, and $\lambda_{\mathrm{S}}=2 \times 10^{-3} \mathrm{~h}^{-1}$, i.e., with probability $q$, they fail with rate $\lambda_{\mathrm{F}}$ and, with probability $1-q$, they fail with rate $\lambda_{\mathrm{S}}$. A server fault is covered with probability 0.9998 . There are three repairmen which repair failed servers at rate $1 \mathrm{~h}^{-1}$. In case the number of failed servers is greater than three, failed servers are selected at random by the repairmen. The measure of interest is the unreliability at time $t, u r(t)$. That measure can be formalized as the $\operatorname{ETRR}(t)$ measure for a CTMC $X$ with state space $S \cup\left\{f_{1}\right\}$, where $S$ includes the states in which the system is operational and $X$ enters the absorbing state $f_{1}$ when the system fails. The states in $S$ of the CTMC can be specified in terms of the number of unfailed servers of each type failing with each rate. The
TABLE 2

Truncation Parameter $K$ in Randomization with Quasistationarity Detection (RQD) and Truncation Parameter $N$ in Standard Randomization (SR) for the Model Outside Class C'

\begin{tabular}{|c|cc|}
\hline \hline$t(\mathrm{~h})$ & $K(\mathrm{RQD})$ & $N(\mathrm{SR})$ \\
\hline \hline 10 & 61 & 77 \\
50 & 228 & 257 \\
100 & 428 & 460 \\
500 & 1,835 & 1,953 \\
1,000 & 3,510 & 3,746 \\
5,000 & 7,528 & 17,682 \\
10,000 & 7,892 & 34,874 \\
50,000 & 8,469 & 171,118 \\
100,000 & 8,664 & 340,699 \\
\hline \hline
\end{tabular}

resulting CTMC has 9,026 states and 65,265 transitions. It is assumed that, initially, the system has all servers unfailed. Then, denoting by $s_{i, j}$ the state in which all servers are unfailed, $i$ servers of type 1 are failing with rate $\lambda_{\mathrm{F}}$ and $j$ servers of type 2 are failing with rate $\lambda_{\mathrm{S}}$, the initial probability distribution of $X$ is

$$
\begin{gathered}
\alpha_{s_{i, j}}=\left(\begin{array}{c}
20 \\
i
\end{array}\right) q^{i}(1-q)^{20-i}\left(\begin{array}{c}
20 \\
j
\end{array}\right) q^{j}(1-q)^{20-j}, \quad 0 \leq i, j \leq 20, \\
\alpha_{k}=0, \quad k \in\left(S-\left\{s_{i, j}, 0 \leq i, j \leq 20\right\}\right) \cup\left\{f_{1}\right\} .
\end{gathered}
$$

Fig. 6 plots $u r(t)$.

The CTMC $X$ has no good "regenerative" state. A reasonable selection for the regenerative state would be the state $s_{i, j}$ which has the largest initial probability. Such a state turns out to be state $s_{2,2}$. Taking that state as the regenerative state made regenerative randomization significantly more costly than standard randomization. Therefore, only results regarding the relative performance of randomization with quasistationarity detection and standard randomization will be given, with a single target time $t$

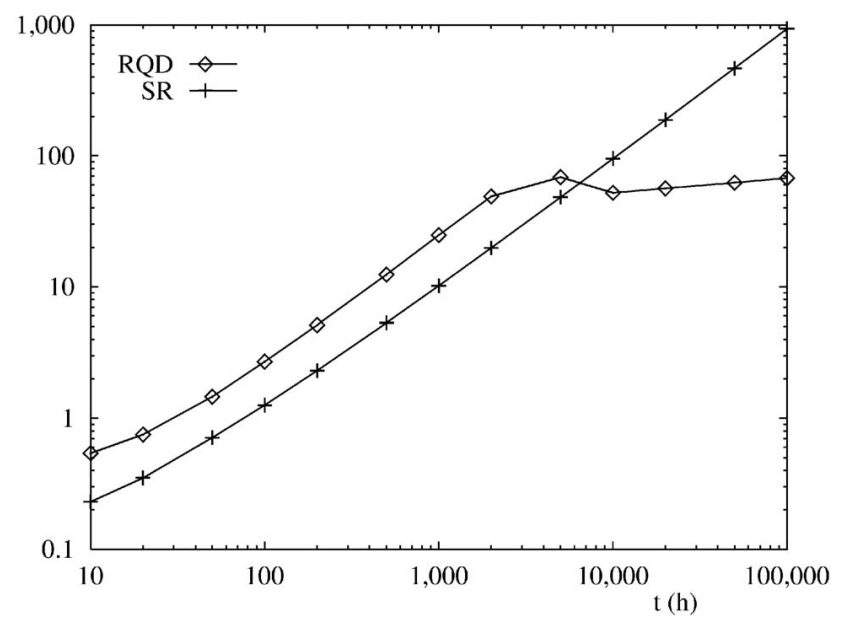

Fig. 7. CPU times in seconds consumed by the methods in seconds for the example outside class $C^{\prime}$ as a function of $t$. 
and a required error $\varepsilon=10^{-10}$ for both methods. Table 2 gives the value of the truncation parameter $K$ in randomization with quasistationarity detection (RQD) and the value of the truncation parameter $N$ in standard randomization (SR) as a function of the time $t$. Fig. 7 gives the corresponding CPU times. We can note that, for large $t$, the truncation parameter $K$ of randomization with quasistationarity detection increases smoothly with $t$ while the truncation parameter $N$ of standard randomization increases approximately linearly with $t$. For large $t$, randomization with quasistationarity detection is significantly faster than standard randomization. Thus, for the largest $t$ considered $(t=100,000 \mathrm{~h}$ ) randomization with quasistationarity detection consumes $67.6 \mathrm{~s}$, whereas standard randomization consumes 930s, making randomization with quasistationarity detection about 14 times faster than standard randomization.

\section{Conclusions}

Exploiting the existence of a quasistationary distribution in the subset of transient states of DTMCs with a certain structure, we have developed a new method, randomization with quasistationarity detection, for the computation of the $\operatorname{ETRR}(t)$ and $\operatorname{EARR}(t)$ measures for a wide class of rewarded CTMCs. The method has the same good properties as the standard randomization method (numerical stability and well-controlled computation error) and, for large stiff models, can be much faster than standard randomization, making possible the analysis of some of these models in affordable CPU times. For class $\mathrm{C}^{\prime}$ models, the method seems to perform slightly worse than regenerative randomization. However, as we have illustrated, the method performs well for models not in class $C^{\prime}$, for which regenerative randomization performs poorly.

\section{RefERENCES}

[1] Handbook of Mathematical Functions, M. Abramowitz and I.A. Stegun, eds. Dover, 1965.

[2] C. Béounes, M. Aguéra, J. Arlat, S. Bachman, C. Bourdeu, J.E. Doucet, K. Kanoun, J.C. Laprie, S. Metge, J. Moreira de Souza, D. Powell, and P. Spiesser, "SURF-2: A Program for Dependability Evaluation of Complex Hardware and Software Systems," Proc. 23rd IEEE Int'l Symp. Fault-Tolerant Computing (FTCS-23), pp. 142150, June 1993.

[3] P.N. Bowerman, R.G. Nolty, and E.M. Schener, "Calculation of the Poisson Cumulative Distribution Function," IEEE Trans. Reliability, vol. 39, no. 2, pp. 158-161, 1990.

[4] J.A. Carrasco, "Transient Analysis of Large Markov Models with Absorbing States Using Regenerative Randomization," Technical Report DMSD_99_2, Universitat Politécnica de Catalunya, Feb. 1999, ftp://ftp-eel.upc.es/techreports.

[5] J.A. Carrasco, "Computation of Bounds for Transient Measures of Large Rewarded Markov Models Using Regenerative Randomization," Computers and Operations Research, vol. 30, pp. 1005-1035, June 2003.

[6] J.A. Carrasco, "Computationally Efficient and Numerically Stable Reliability Bounds for Repairable Fault-Tolerant Systems," IEEE Trans. Computers, vol. 51, no. 3, pp. 254-268, Mar. 2002.

[7] J.A. Carrasco, "Markovian Dependability/Performability Modeling of Fault-Tolerant Systems," Reliability Eng. Handbook, H. Pham, ed., pp. 613-642, Springer-Verlag, 2003.

[8] G. Ciardo, J. Muppala, and K. Trivedi, "SPNP: Stochastic Petri Net Package," Proc. Third IEEE Int'l Workshop Petri Nets and Performance Models (PNPM89), pp. 142-151, Dec. 1989.

[9] B.L. Fox and P.W. Glynn, "Computing Poisson Probabilities," Comm. ACM, vol. 31, pp. 440-445, 1988.
[10] A. Goyal, W.C. Carter, E. de Souza e Silva, and S.S. Lavenberg, "The System Availability Estimator," Proc. 16th IEEE Int'l Symp. Fault-Tolerant Computing (FTCS-16), pp. 84-89, July 1986.

[11] W.K. Grassmann, "Transient Solutions in Markovian Queuing Systems," Computers and Operations Research, vol. 4, pp. 47-53, 1977.

[12] W.K. Grassmann, "Means and Variances of Time Averages in Markovian Environments," European J. Operational Research, vol. 31, no. 4, pp. 839-854, Oct. 1984.

[13] D. Gross and D.R. Miller, "The Randomization Technique as a Modelling Tool and Solution Procedure for Transient Markov Processes," Operations Research, vol. 32, pp. 343-361, 1984.

[14] M. Kijima, Markov Processes for Stochastic Modeling. London: Chapman \& Hill, 1997.

[15] L. Knüsel, "Computation of the Chi-Square and Poisson Distribution," SIAM J. Scientific and Statistical Computing, vol. 7, no. 3, pp. 1022-1036, July 1986

[16] M. Malhotra, J.K. Muppala, and K.S. Trivedi, "Stiffness-Tolerant Methods for Transient Analysis of Stiff Markov Chains," Microelectronics and Reliability, vol. 34, no. 11, pp. 1825-1841, 1994.

[17] M. Malhotra, "A Computationally Efficient Technique for Transient Analysis of Repairable Markovian Systems," Performance Evaluation, nos. 1-2, pp. 311-331, Nov. 1995.

[18] B. Melamed and M. Yadin, "Randomization Procedures in the Computation of Cumulative-Time Distributions over Discrete State Markov Processes," Operations Research, vol. 32, pp. 926-944, 1984.

[19] B. Melamed and M. Yadin, "Numerical Computation of SojournTime Distributions in Queuing Networks," J. ACM, vol. 31, no. 4, pp. 839-854, Oct. 1984.

[20] D.R. Miller, "Reliability Calculation Using Randomization for Markovian Fault-Tolerant Computing Systems," Proc. 13th IEEE Int'l Symp. Fault-Tolerant Computing (FTCS-13), pp 284-289, June 1983.

[21] A.P. Moorsel and W.H. Sanders, "Adaptive Uniformization," Comm. in Statistics-Stochastic Models, vol. 10, no. 3, pp. 619-647, 1994.

[22] A.P. Moorsel and W.H. Sanders, "Transient Solution of Markov Models by Combining Adaptive and Standard Uniformization," IEEE Trans. Reliability, vol. 46, no. 3, pp. 430-440, 1997.

[23] A. Reibman and K.S. Trivedi, "Numerical Transient Analysis of Markov Models," Computers and Operations Research, vol. 15, pp. 19-36, 1988.

[24] A. Reibman and K. Trivedi, "Transient Analysis of Cumulative Measures of Markov Model Behavior," Comm. in Statistics-Stochastic Models, vol. 5, no. 4, pp. 683-710, 1989.

[25] S.M. Ross, Stochastic Processes. John Wiley \& Sons, 1983

[26] W.H. Sanders, W.D. Obal II, M.A. Qureshi, and F.K. Widjanarko, "The UltraSAN Modeling Environment," Performance Evaluation, vol. 24, nos. 1-2, pp. 89-115, 1995.

[27] B. Sericola, "Availability Analysis of Repairable Computer Systems and Stationarity Detection," IEEE Trans. Computers, vol. 48, no. 11, pp. 1166-1172, Nov. 1999.

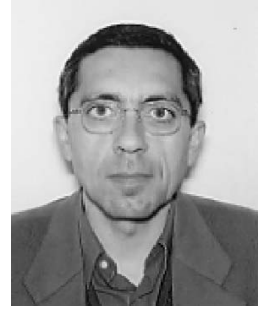

Juan A. Carrasco received the Engineer degree in industrial engineering from the Polytechnical University of Catalonia (UPC) in 1982, the MSc degree in computer science from Stanford University in 1987, and the PhD degree in industrial engineering from UPC, also in 1987. $\mathrm{He}$ is currently an associate professor in the Electronics Engineering Department of UPC in Barcelona, Spain. He held a visiting position at INRIA (CNRS), Rennes, France, in 1996, working on bounding methods for Markovian dependability models and "onthe-fly" model solution techniques. His current research interests encompass methodologies for the modeling and evaluation of faulttolerant systems, including bounding methods, numerical techniques, and combinatorial methods, topics in which he has published more than 50 papers in refereed journals and conference proceedings. Many of those methodologies have been incorporated in METFAC-2, a Markovian modeling tool developed under his direction. He has been the principal investigator of several research projects funded by public and private institutions and has been on the program committees of several international conferences. He is a senior member of the IEEE and the IEEE Computer Society. 